\title{
Analysis and Results from a Flush Airdata Sensing System in Close Proximity to Firing Rocket Nozzles
}

\author{
Aliyah N. Ali ${ }^{1}$ \\ Dryden Flight Research Center, Edwards, California, 93524 \\ Jerry L. Borrer ${ }^{2}$ \\ Johnson Space Center, Houston, Texas, 77058
}

This paper presents information regarding the nosecap Flush Airdata Sensing (FADS) system on Orion's Pad Abort 1 (PA-1) vehicle. The purpose of the nosecap FADS system was to test whether or not useful data could be obtained from a FADS system if it was placed in close proximity to firing rocket nozzles like the Attitude Control Motor (ACM) nozzles on the PA-1 Launch Abort System. The nosecap FADS system used pressure measurements from a series of pressure ports which were arranged in a cruciform pattern and flush with the surface of the vehicle to estimate values of angle of attack, angle of sideslip, Mach number, impact pressure, and freestream static pressure. This paper will present the algorithms employed by the FADS system along with the development of the calibration datasets and a comparison of the final results to the Best Estimated Trajectory (BET) data for PA-1. Also presented in this paper is a Computational Fluid Dynamics (CFD) study to explore the impact of the ACM on the nosecap FADS system. The comparison of the nosecap FADS system results to the BET and the CFD study showed that more investigation is needed to quantify the impact of the firing rocket motors on the FADS system.

$\begin{array}{ll}\text { ACM } & =\text { Attitude Control Motor } \\ \text { AM } & =\text { Abort Motor } \\ \text { BET } & =\text { Best Estimated Trajectory } \\ \mathrm{C}_{\mathrm{p}} & =\text { surface pressure coefficient } \\ \text { CEV } & =\text { Crew Exploration Vehicle } \\ \text { CFD } & =\text { Crew Module } \\ \text { CM } & =\text { Flush Airdata Sensing System } \\ \text { FADS } & =\text { Forward Bay Cover } \\ \text { FBC } & =\text { Flight Test Article } \\ \text { FTA } & =\text { Jettison Motor } \\ \text { JM } & =\text { Launch Abort System } \\ \text { LAS } & =\text { Launch Abort Vehicle } \\ \text { LAV } & =\text { estimation algorithm geometry matrix } \\ \text { M } & =\text { initial Mach guess } \\ M_{\text {guess }} & =\text { freestream Mach number } \\ \text { M }_{\infty} & =\text { total number of pressure ports } \\ n & =\text { National Aeronautics and Space Administration } \\ \text { NASA } & =\text { Operational Flight Instrumentation } \\ \text { OFI } & =\text { freestream static pressure, psf } \\ \mathrm{P}_{\infty} & \end{array}$

\footnotetext{
${ }^{1}$ Aerospace Engineer, Aerodynamics and Propulsion Branch, M/S 4800-2108, AIAA Senior Member.

${ }^{2}$ MPCV Aerodynamics Flight Test Lead, Applied Aerosciences and CFD Branch, M/S EG3, AIAA non-member.
} 


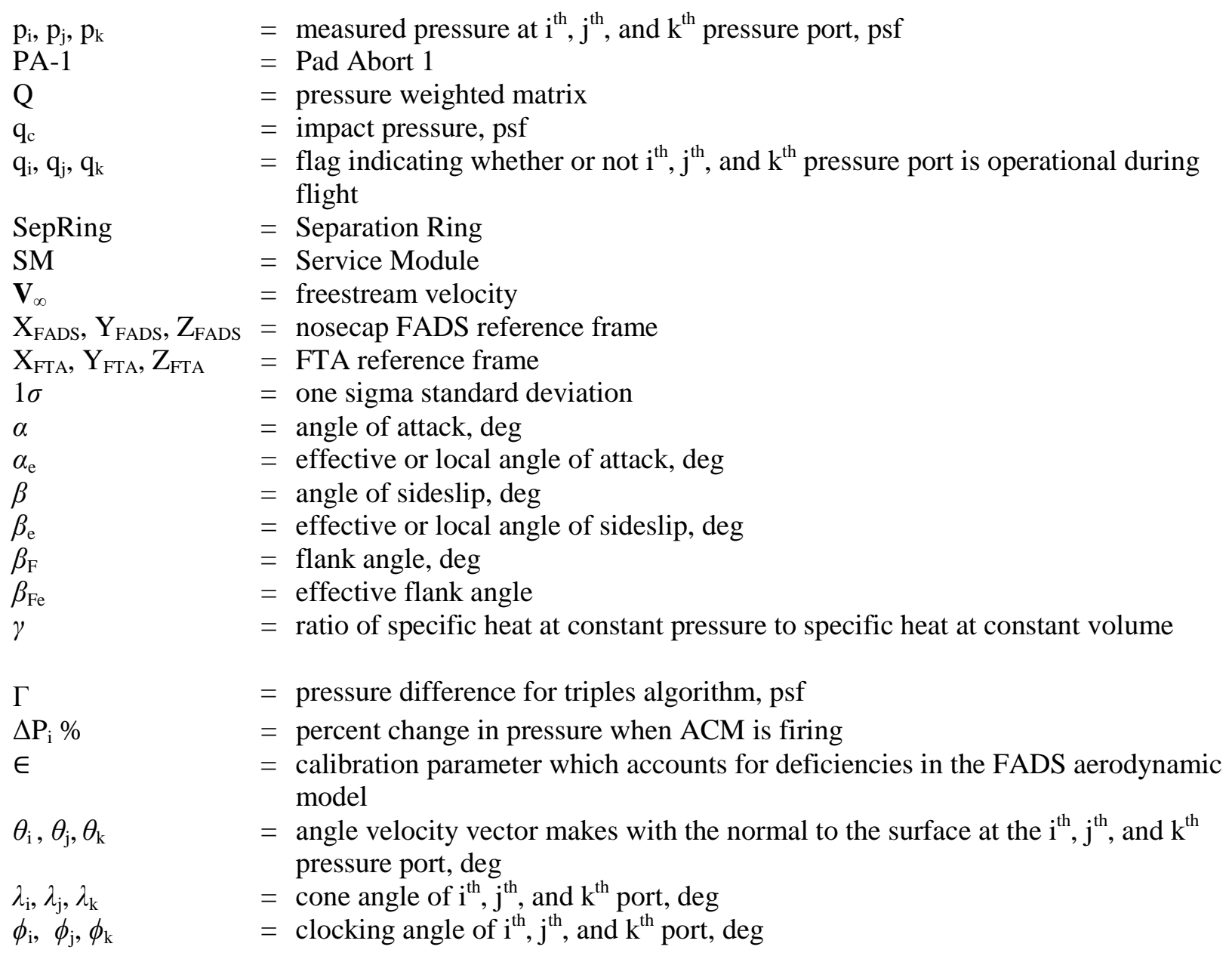

\section{Introduction}

Zlush airdata sensing (FADS) systems were developed to facilitate nonintrusive determination of I airdata during flight. Unlike airdata booms and probes that penetrate the flow away from the influence of the vehicle, FADS systems use values of surface pressures measured by pressure sensors mounted flush with the surface to estimate angle of attack $(\alpha)$, angle of sideslip $(\beta)$, impact pressure $\left(\mathrm{q}_{\mathrm{c}}\right)$, freestream static pressure $\left(\mathrm{P}_{\infty}\right)$, and Mach number. FADS systems build on work started in the early 1960's with the X-15 (North American Aviation, now The Boeing Company, Chicago, Illinois) program ${ }^{1}$ and have performed successfully on vehicles like the X-43A (Micro Craft Incorporated, Tullahoma, Tennessee). ${ }^{2}$ Also, the Real-Time Flush Airdata Sensing (RT-FADS) system flown on the National Aeronautics and Space Administration (NASA)'s F-18 (McDonnell Douglas, now The Boeing Company, Chicago, Illinois) research aircraft has shown positive results. ${ }^{3}$

There were two FADS systems on the Pad Abort 1 (PA-1) vehicle. One was located on the LAS nosecap while the other was located on the Crew Module (CM) heatshield. ${ }^{4}$ This paper will cover the details of the nosecap FADS system.

\section{A. PA-1 Vehicle Description}

The PA-1 vehicle, whose basic layout is illustrated in Fig. 1, was successfully launched from the U.S. Army's White Sands Missile Range in New Mexico, on May 6, 2010. PA-1 was the first in a sequence of atmospheric flight tests necessary for the development of the Orion Crew Exploration Vehicle (CEV) which was a component of the now deactivated Constellation, a human space flight project. Orion was comprised of three modules: the Crew Module (CM), the Service Module (SM), and the Launch Abort System (LAS). The CM was intended to house the astronauts while the SM, which was not a part of the 
PA-1 test, was intended to provide power and propulsion to the spacecraft while in orbit. The LAS had the capability to abort from the launch pad as well as during ascent at medium and high altitudes.

Figure 1 highlights the major components of the PA-1 vehicle. The Separation Ring (SepRing) remained on the launch pad after launch. As can be seen in Fig. 2, the LAS consisted of three active motors: the Abort Motor (AM), the Jettison Motor (JM), and the Attitude Control Motor (ACM). The AM was a solid rocket motor with four reverse flow nozzles canted outward at $25^{\circ}$ from the motor centerline. The AM generated the thrust necessary to pull the vehicle away from the SepRing which remained on the launch pad for the duration of the PA-1 test. The AM provided a maximum thrust of approximately $5 \mathrm{x}$ $10^{5} \mathrm{lbf}^{5}$

The JM was a solid rocket motor with four scarfed nozzles canted at $35^{\circ}$ from the motor centerline. The JM was intended to pull the LAS away from the CM. The JM was capable of producing a maximum thrust of approximately $4.0 \times 10^{4}$ lbf. $^{5}$

The ACM was a solid rocket motor with eight radially spaced, throttled nozzles placed at $45^{\circ}$ increments symmetrically about the LAS centerline. The ACM provided directional control for the LAS and provided approximately $6.5 \times 10^{3} \mathrm{lbf}$ of commanded omnidirectional thrust during the first $7 \mathrm{~s}$ of flight, followed by $2.5 \times 10^{3} \mathrm{lbf}$ of commanded omnidirectional thrust during the next $20 \mathrm{~s}$ of flight. ${ }^{6}$

Figures 3 and 4 show the PA-1 LAS and CM at two different points along the trajectory. Figure 3 shows the AM and ACM firing during the initial ascent of the vehicle, and Fig. 4 shows the LAS moving away from the CM after LAS jettison with the ACM and JM firing.

\section{B. PA-1 Flight Sequence}

The PA-1 flight sequence was timer based and is illustrated in Fig. 5. A summary of the critical events is given in Table 1. When the pad abort was initiated, the Launch Abort Vehicle (LAV) was pulled away from the pad by the AM, a pitch-over sequence was initiated for downrange distance, and the vehicle was stabilized by the ACM. The AM burned out after $2.68 \mathrm{~s}$, and the ACM continued to stabilize the vehicle. Then the ACM began to reorient and re-stabilize the LAV with the heatshield forward in preparation for separation of the LAS from the CM. After reorientation was complete at $17.04 \mathrm{~s}$, the JM fired to separate the CM from the LAS at 21.03 s. Then the Forward Bay Cover (FBC) at the top of the $\mathrm{CM}$ was jettisoned to begin the parachute deployment sequence with the drogue parachutes being deployed at $24.26 \mathrm{~s}$ followed by the main parachutes at $33.61 \mathrm{~s}$. The CM touched down at $134.36 \mathrm{~s}$ after launch.

Table 1. PA-1 major trajectory events.

\begin{tabular}{ccr}
\hline \hline Event number & Description & Time, s \\
\hline 1 & Launch & 0.00 \\
2 & Abort Motor burnout & 2.68 \\
3 & Reorientation started & 10.27 \\
4 & Reorientation completed & 17.04 \\
5 & LAS jettison & 21.03 \\
6 & FBC jettison & 22.17 \\
7 & Drogue parachute deployment & 24.26 \\
8 & Main parachute deployment & 33.61 \\
9 & LAS touchdown & 47.85 \\
10 & Main parachute full inflation & 50.35 \\
11 & CM touchdown & 134.36 \\
\hline \hline
\end{tabular}




\section{PA-1 Nosecap FADS System}

The purpose of the nosecap FADS system on PA-1 was to test whether or not useful data could be obtained from this system given the environment it would be subjected to. When the decision was made to install a FADS system on the PA-1 LAS nosecap, FADS systems had not been located in such close proximity to firing rocket nozzles like the nosecap FADS system had been (Fig. 6).

The nosecap FADS system gathered pressure data during flight, and this data was post-processed to estimate flight airdata parameters. Parameters were estimated from launch until the start of vehicle reorientation. The portion of the flight trajectory covered by the nosecap FADS system and the heatshield FADS system ${ }^{4}$ is shown in Fig. 5. Neither FADS system produced useful data during vehicle re-orientation because they were designed to generate airdata parameters during nose forward flight of the LAS and heatshield forward flight of the CM with angle of attack and sideslip not exceeding $20^{\circ}$ for the LAS and $40^{\circ}$ for the CM.

The nosecap FADS system consisted of 9 absolute pressure transducers arranged in a cruciform pattern. These sensors had a range of 0-15 psia and took pressure samples at a rate of 101.73 samples per second. The sensors were mounted flush with the surface of the nosecap. Since the pressure sensors were located at the ports and there was no pressure tubing involved, lag in the system was not a concern.

As can be seen from Fig. 6, the nosecap FADS pressure port matrix was approximately 48 inches away from the ACM nozzles. Since the PA-1 flight regime was subsonic, it was inferred that there might be enough influence of the ACM on the FADS pressure ports to have significant impact on the resulting FADS airdata parameter estimates. Some CFD analysis was done to quantify this influence of the ACM's on the FADS system output. This analysis will be discussed after the results from the nosecap FADS system for the PA-1 trajectory are presented.

\section{Frames of Reference and Angle Definitions}

The final data set produced by each FADS system referenced the Flight Test Article (FTA) reference frame, which is illustrated in Fig. 7. As can be seen in Fig. 7, the $\mathrm{X}_{\mathrm{FTA}}$ axis goes through the center of the vehicle from nose to tail.

Also shown in Fig. 7 are angle of attack $(\alpha)$, angle of sideslip $(\beta)$, and flank angle $\left(\beta_{\mathrm{F}}\right)$. Angle of attack is defined as the angle between the $X$-axis and the projection of the velocity vector $\left(\mathbf{V}_{\infty}\right)$ onto the $\mathrm{XZ}$ plane. Angle of sideslip $(\beta)$ is defined as the angle between $\mathbf{V}_{\infty}$ and the projection of $\mathbf{V}_{\infty}$ onto the $\mathrm{XZ}$ plane. Flank angle $\left(\beta_{\mathrm{F}}\right)$ is defined as the angle between the $\mathrm{X}$-axis and the projection of $\mathbf{V}_{\infty}$ onto the $\mathrm{XY}$ plane. The direction of positive $\alpha, \beta$, and $\beta_{\mathrm{F}}$ is illustrated in Fig. 7. Sideslip is related to $\beta_{\mathrm{F}}$ and $\alpha$ by Eq. (1).

$$
\beta=\tan ^{-1}\left(\tan \left(\beta_{F}\right) \times \cos (\alpha)\right)
$$

Figure 8 is an illustration of the nosecap FADS ports and the nosecap FADS reference frame relative to the FTA reference frame. Figure 8 is a nose to tail view. It shows the nosecap FADS ports and the nosecap FADS reference frame clocked $30^{\circ}$ clockwise of the FTA reference frame. The $\mathrm{X}_{\mathrm{FADs}}$ axis and the $\mathrm{X}_{\mathrm{FTA}}$ axis go into the page. All calculations for the FADS system referenced the FADS reference frame. The final answers were transformed to the FTA reference frame.

Figure 9 illustrates the definition of clocking angle $(\phi)$ and cone angle $(\lambda)$. These angles are used to describe the locations of the FADS ports on the vehicle. Clocking angle is defined as the angle between the negative $\mathrm{Z}$-axis and a line drawn through the port which intersects and is normal to the $\mathrm{X}$-axis. Cone angle is defined as the angle which the normal to the port surface makes with the X-axis. Clocking angle and cone angle are illustrated in Fig. 9. The values of $\phi$ and $\lambda$, which are used in the FADS algorithms, referenced the FADS reference frame illustrated in Fig. 8. Since the FADS ports were aligned with the FADS reference frame, the values of $\phi$ for the FADS ports were $0^{\circ}, 90^{\circ}, 180^{\circ}$, or $270^{\circ}$ respectively. 


\section{E. Flush Airdata Sensing Pressure Model}

The foundation of a FADS system is that airdata states can be estimated from surface pressure measurements. To perform this estimation, an aerodynamic model is needed which could capture the salient features in the flow and be valid over a wide range of Mach numbers. The aerodynamic model given by Eq. (2) was found to be most applicable. ${ }^{8}$

$$
p_{i}=q_{c}\left[\cos ^{2}\left(\theta_{i}\right)+\in \sin ^{2}\left(\theta_{i}\right)\right]+P_{\infty}
$$

In Eq. (2), $\mathrm{p}_{\mathrm{i}}$ represents pressure measured at the $\mathrm{i}^{\text {th }}$ port, $\mathrm{q}_{\mathrm{c}}$ represents impact pressure, $\mathrm{P}_{\infty}$ represents freestream static pressure, and $\in$ is a calibration parameter. $\theta_{\mathrm{i}}$ is defined as the angle that the velocity vector makes with the normal to the surface at the $\mathrm{i}^{\text {th }}$ port. It is related to $\left(\alpha_{\mathrm{e}}\right)$, the effective or local angle of attack and $\left(\beta_{\mathrm{e}}\right)$, the effective or local angle of sideslip, by Eq. (3) ${ }^{8}$ where $\phi_{\mathrm{i}}$ and $\lambda_{\mathrm{i}}$ represent the clock and cone angles of the $\mathrm{i}^{\text {th }}$ port.

$$
\begin{aligned}
\cos \left(\theta_{i}\right) & =\cos \left(\alpha_{e}\right) \cos \left(\beta_{e}\right) \cos \left(\lambda_{i}\right) \\
& +\sin \left(\beta_{e}\right) \sin \left(\phi_{i}\right) \sin \left(\lambda_{i}\right) \\
& +\sin \left(\alpha_{e}\right) \cos \left(\beta_{e}\right) \cos \left(\phi_{i}\right) \sin \left(\lambda_{i}\right)
\end{aligned}
$$

Eq. (2) is a combination of the closed form potential flow solution for a blunt body and the modified Newtonian flow model ${ }^{8}$. To blend these two solutions over a wide range of Mach numbers, $\in$ was used. The calibration parameter, $\in$, also accounts for deficiencies in the FADS aerodynamic model. These deficiencies include the steady state effects of side-wash and up-wash of the vehicle on the FADS system ports.

From Eqs. (2) and (3), a minimum of five pressure ports are necessary since there are four airdata states and one calibration parameter to be estimated. It has been found, however, that using five ports results in a system which is sensitive to noise. ${ }^{8}$ To reduce noise sensitivity and increase port redundancy, more than five pressure ports are necessary. The nosecap FADS system had nine ports.

\section{F. Calculation of Angle of Attack and Flank Angle}

The "Triples Algorithm" ${ }^{8}$ is used to estimate $\alpha_{\mathrm{e}}$. This algorithm takes the differences of three distinct surface pressures defined by Eq. (2), resulting in the elimination of the pressure related states and the calibration parameter $\left(\mathrm{q}_{c}, \mathrm{P}_{\infty}\right.$, and $\in$ ). The resulting pressure equation is given in Eq. (4):

$$
\Gamma_{i k} \cos ^{2} \theta_{j}+\Gamma_{j i} \cos ^{2} \theta_{k}+\Gamma_{k j} \cos ^{2} \theta_{i}=0
$$

where as shown in Eq. (5),

$$
\begin{gathered}
\Gamma_{i k}=p_{i}-p_{k} \\
\Gamma_{j i}=p_{j}-p_{i} \\
\Gamma_{k j}=p_{k}-p_{j}
\end{gathered}
$$

The pressures at the $i^{\text {th }}, j^{\text {th }}$, and $k^{\text {th }}$ ports are given by $p_{i}, p_{j}$, and $p_{k}$. The angle between the velocity vector and the normal to the surface at the $\mathrm{i}^{\text {th }}, \mathrm{j}^{\text {th }}$, and $\mathrm{k}^{\text {th }}$ ports are given by $\theta_{\mathrm{i}}, \theta_{\mathrm{j}}$, and $\theta_{\mathrm{k}}$.

The layout of the pressure ports in a cruciform pattern allowed for calculations for $\alpha_{\mathrm{e}}$ to be decoupled from $\beta_{\mathrm{e}}$, causing $\beta_{\mathrm{e}}$ to fall out of Eq. (4). The resulting solution for $\alpha_{\mathrm{e}}$ is given by Eq. (6): 


$$
2 \alpha_{e}=\tan ^{-1}\left(\frac{A}{B}\right)
$$

where A and B are defined by Eq. (7):

$$
\begin{gathered}
A=\Gamma_{i k} \sin ^{2}\left(\lambda_{j}\right)+\Gamma_{j i} \sin ^{2}\left(\lambda_{k}\right)+\Gamma_{k j} \sin ^{2}\left(\lambda_{i}\right) \\
B=\Gamma_{i k} \cos \left(\phi_{j}\right) \sin \left(\lambda_{j}\right) \cos \left(\lambda_{j}\right) \\
+\Gamma_{j i} \cos \left(\phi_{k}\right) \sin \left(\lambda_{k}\right) \cos \left(\lambda_{k}\right) \\
+\Gamma_{k j} \cos \left(\phi_{i}\right) \sin \left(\lambda_{i}\right) \cos \left(\lambda_{i}\right)
\end{gathered}
$$

and from Eq. (6), if $2 \alpha_{\mathrm{e}}$ was greater than $\pi / 2$ then $\alpha_{\mathrm{e}}$ was given by Eq. (8):

$$
\alpha_{e}=\frac{\pi-2 \alpha_{e}}{2}
$$

or, if $2 \alpha_{\mathrm{e}}$ was less than $-\pi / 2$, then $\alpha_{\mathrm{e}}$ was given by Eq. (9):

$$
\alpha_{e}=\frac{-\left(\pi+2 \alpha_{e}\right)}{2}
$$

The ports lying along the Z-axis were used to determine $\alpha_{\mathrm{e}}$. All the combinations of three distinct ports were used, and the overall average of the resulting $\alpha_{\mathrm{e}}$ from each triple was found.

By definition, $\beta_{\mathrm{F}}$ is orthogonal to $\alpha$ and since the FADS ports were distributed in a cruciform pattern, the triples algorithm could be used to calculate effective flank angle $\left(\beta_{\mathrm{Fe}}\right)$ by using the ports on the $\mathrm{Y}_{\mathrm{FADS}}$ axis. To calculate $\beta_{\mathrm{Fe}}$ with the triples algorithm, a $90^{\circ}$ counterclockwise rotation was applied to the clocking angles of the ports on the $\mathrm{Y}_{\mathrm{FADS}}$ axis then the same procedure as was followed for calculating $\alpha_{\mathrm{e}}$.

\section{G. Calculating $\in$}

The calibration parameter, $\in$ accounts for deficiencies in the FADS aerodynamic model. These deficiencies include the steady state effects of side-wash and up-wash of the vehicle on the FADS ports. The effects of the ACM firing were not accounted for. For the PA-1 nosecap FADS, $\in$ was calculated by finding the least squares solution, with respect to $\in$, of the FADS aerodynamic model for a given set of $n$ FADS ports at a given flight condition.

From Eq. (1), which defines the pressure at each pressure port, the complete set of equations defining the pressures at each pressure port on the right and the measured pressures on the left are given by Eq. (10). 


$$
\left[\begin{array}{c}
p_{1} \\
\cdot \\
\cdot \\
p_{n}
\end{array}\right]=\left[\begin{array}{cc}
\left(\cos ^{2}\left(\theta_{1}\right)+\in \sin ^{2}\left(\theta_{1}\right)\right) & 1 \\
\cdot & \cdot \\
\left.\cdot \sin ^{2}\left(\theta_{n}\right)\right) & 1
\end{array}\right]\left[\begin{array}{l}
q_{c} \\
P_{\infty}
\end{array}\right]
$$

Applying least squares to Eq. (10) yields Eq. 11.

$$
\epsilon=\frac{\sum_{i=1}^{n} \sin ^{2} \theta_{i}\left(C_{p}-\cos ^{2} \theta_{i}\right)}{\sum_{i=1}^{n} \sin ^{4} \theta_{i}}
$$

In Eq. (11), $C_{p}$ is the pressure coefficient defined by Eq. (12).

$$
C_{p}=\frac{p_{i}-P_{\infty}}{q_{c}}
$$

\section{H. Mach Number, Impact Pressure, and Static Pressure Calculation}

After $\alpha_{\mathrm{e}}$ and $\beta_{\mathrm{e}}$ are calculated, the parameters $\mathrm{q}_{\mathrm{c}}$ and $\mathrm{P}_{\infty}$ were extracted using Eq. (13) which is the iterative estimator derived by Whitmore: ${ }^{8}$

$$
\left[\begin{array}{l}
q_{c} \\
P_{\infty}
\end{array}\right]_{(j+1)}=\left\{\left[M_{(j)}^{T} Q M_{(j)}\right]^{-1} M_{(j)}^{T} Q\right\}\left[\begin{array}{c}
p_{1} \\
\cdot \\
\cdot \\
\cdot \\
p_{n}
\end{array}\right]
$$

From Eq. (13), $\mathrm{M}_{(\mathrm{j})}$ is defined by Eq. (14), and Q is defined by Eq. (15).

$$
\begin{aligned}
& M_{(j)}=\left[\begin{array}{cc}
\left(\cos ^{2}\left(\theta_{1}\right)+\epsilon_{(j)} \sin ^{2}\left(\theta_{1}\right)\right) & 1 \\
\cdot & \\
\cdot & \\
\left(\cos ^{2}\left(\theta_{n}\right)+\epsilon_{(j)} \sin ^{2}(n)\right) & 1
\end{array}\right] \\
& Q=\left[\begin{array}{ccc}
q_{1} & \ldots & 0 \\
\cdot & \cdot & \cdot \\
\cdot & \cdot & \cdot \\
\cdot & & \cdot \\
0 & . & q_{n}
\end{array}\right]
\end{aligned}
$$

In Eqs. (13) and 14, the subscript (j) refers to the result of the $\mathrm{j}^{\text {th }}$ iteration of the estimator. In Eq. (15), $\mathrm{q}_{1}$ thru $\mathrm{q}_{n}$ are flags for each of the pressure ports 1 thru $n$. These flags take on a value of 1 if the port is operational or 0 if the port is not operational during flight. For PA-1, all ports were operational.

With $\mathrm{q}_{\mathrm{c}}$ and $\mathrm{P}_{\infty}$, Mach number $\left(\mathrm{M}_{\infty}\right)$ could be calculated from the isentropic flow relation for subsonic flow as shown in Eq. (16). 


$$
\frac{q_{c}}{P_{\infty}}=\left(1+\frac{\gamma-1}{2} M_{\infty}^{2}\right)^{\frac{\gamma}{\gamma-1}}-1
$$

Smoothing of the final set of FADS system data was done with a moving average filter. Correcting the values of $\alpha$ and $\beta$ generated by the FADS system for rotation rates of the vehicle was not performed.

\section{Calibration Data Set}

The nosecap FADS system relied on a calibration database made up entirely of Computational Fluid Dynamics (CFD) data. This data was generated with the use OVERFLOW, ${ }^{9}$ a Navier-Stokes flow solver for structured grids. Only the portion of the vehicle forward of the AM nozzles was modeled. The ACM and the JM were not modeled. The range of Mach, $\alpha$ and $\beta$ covered by the original database is listed in Table 2. This database was expanded in $\beta$ given that the portion of the vehicle, which was modeled, was axisymmetric. The complete set of alpha and beta covered by the calibration database is illustrated in Fig. 10. This calibration database serves the purpose of accounting for phenomena not modeled in the governing aerodynamic equation for the FADS system except the firing motors.

Table 2. Range of CFD solutions used to build nosecap FADS calibration data set.

\begin{tabular}{cc}
\hline \hline Parameter & Range \\
\hline Mach & $0.2,0.4,0.5,0.6,0.7$ \\
$\alpha$ & $0.0,2.5,5.0,7.5,10,12.5,15,20$ \\
$\beta$ & 0.0 \\
\hline
\end{tabular}

Using the available CFD data, a numerical model was generated to predict free stream $\alpha$ and $\beta_{\mathrm{F}}$ from $\alpha_{\mathrm{e}}, \beta_{\mathrm{Fe}}$, and Mach number. An illustration of the calibration data set for $\alpha$ at zero $\beta_{\mathrm{Fe}}$ is illustrated in Fig. 11. As can be seen from Fig. 11, as one moves away from zero $\alpha_{\mathrm{e}}$, the dependence of $\alpha$ on Mach number increases. Since $\alpha$ and $\beta_{\mathrm{F}}$ are orthogonal and the portion of the vehicle modeled in the CFD calibration data was axisymmetric, the calibration tables for $\alpha$ and $\beta_{\mathrm{F}}$ were the same.

Like $\alpha$ and $\beta_{\mathrm{F}}$, $\in$ was also a function of $\alpha_{\mathrm{e}}, \beta_{\mathrm{Fe}}$, and Mach number. The variation of $\in$ with respect to $\alpha_{\mathrm{e}}$ and Mach number at zero $\beta_{\mathrm{Fe}}$ is illustrated in Fig. 12. Again, since $\alpha$ and $\beta_{\mathrm{F}}$ are contained in orthogonal planes and the portion of the vehicle which was modeled in the CFD was axisymmetric, the plot of the variation of $\epsilon$ with respect to $\beta_{\mathrm{Fe}}$ at zero $\alpha_{\mathrm{e}}$ is the same as the plot shown in Fig. 12.

\section{J. Algorithm Flow}

Figure 13 illustrates the implementation of the nosecap FADS algorithm. First, the triples algorithm used the flight pressure data $\left(\mathrm{p}_{\mathrm{i}}\right)$ to calculate $\alpha_{\mathrm{e}}$ and $\beta_{\mathrm{Fe}}$. Then these values along with an initial guess of Mach number ( $\mathrm{M}_{\text {guess }}$ ) were used to calculate $\in$ from the $\in$ calibration tables. The calibration parameter, $\mathrm{M}_{\text {guess }}, \mathrm{p}_{\mathrm{i}}, \alpha_{\mathrm{e}}$, and $\beta_{\mathrm{Fe}}$ were then used in the first iteration of Eq. (13). If the difference between the resulting $\mathrm{M}_{\infty}$ and $\mathrm{M}_{\text {guess }}$ from the first iteration were greater than the tolerance value of $1 \times 10^{-4}$ then $\mathrm{M}_{\infty}, \mathrm{p}_{\mathrm{i}}$, $\alpha_{\mathrm{e}}$, and $\beta_{\mathrm{Fe}}$ were used to calculate another value of $\epsilon$ and this value was used along with $\mathrm{M}_{\infty}, \alpha_{\mathrm{e}}$, and $\beta_{\mathrm{Fe}}$ in a second iteration of Eq. (13). The previously mentioned procedure was repeated until the difference between $\mathrm{M}_{\infty}$ from the current iteration and $\mathrm{M}_{\infty}$ from the previous iteration was less than the tolerance value of $1 \times 10^{-4}$. Then $\mathrm{M}_{\infty}, \alpha_{\mathrm{e}}$, and $\beta_{\mathrm{Fe}}$ were used to determine $\alpha$ and $\beta_{\mathrm{F}}$ from their respective calibration tables. And $\alpha$ and $\beta_{\mathrm{F}}$ were used to calculate $\beta$. Finally, $\alpha$ and $\beta$ were transformed from the FADS coordinate frame to the FTA coordinate frame.

\section{K. Results}

The final set of values for $\alpha, \beta, \mathrm{q}_{\mathrm{c}}, \mathrm{M}_{\infty}$, and $\mathrm{P}_{\infty}$ were compared to the best estimated trajectory (BET) ${ }^{8}$ in order to gauge the performance of the FADS system. The BET used inertial data, radar tracking, optical 
observations, and the day of flight atmosphere profile to determine the air data parameters of the vehicle along its trajectory. The BET was the most reliable source of data available for comparison.

Figures 14 to 18 show comparisons of the FADS data to the BET. Each figure has two plots. The first plot illustrates the FADS parameter of interest along with the corresponding BET parameter and the BET one sigma standard deviation $(1 \sigma)$. The second plot illustrates the difference between the BET and the FADS data. Both plots show the portion of the trajectory for which the nosecap FADS system was calibrated. The points in time when the abort motor burned out and when reorientation started are marked by green vertical lines in both plots.

Figure 14 shows how $\alpha$ from the FADS compares to the BET. As can be seen in this figure, both sets of data followed the same trends and FADS data lay outside of the BET $1 \sigma$ for much of the time. After about $0.5 \mathrm{~s}$ after launch to just after vehicle reorientation, the FADS data differed from the BET by a maximum of $2^{\circ}$.

Figure 15 shows how the FADS predicted $\beta$ differed from the BET. As can be seen in Fig. 15, the FADS and the BET followed similar trends, but the FADS data lay outside the BET $1 \sigma$ throughout this portion of the trajectory. On average, the difference observed for $\beta$ was larger than the difference observed for $\alpha$. Given that $\beta$ is a combination of $\beta_{\mathrm{F}}$ and $\alpha$, the larger difference observed for $\beta$ could possibly be due to the combination of the inherent errors in $\beta_{\mathrm{F}}$ and $\alpha$.

Figure 16 shows how $\mathrm{P}_{\infty}$ predicted by the FADS system differed from the BET prediction. Freestream static pressure predicted by the FADS was expected to be higher than the BET due to the proximity of the FADS pressure ports to the firing ACM nozzles. More analysis needs to be performed to understand why $\mathrm{P}_{\infty}$ predicted by the FADS system followed a different trend than the BET during the first $2.5 \mathrm{~s}$ of flight, and why they differed in magnitude by as much as 120 psf.

Figure 17 shows how $\mathrm{q}_{\mathrm{c}}$ predicted by the nosecap FADS system differed from the BET prediction. The BET and the FADS data followed similar trends, and the FADS system continuously under predicted $\mathrm{q}_{\mathrm{c}}$. As can be seen in Fig. 17, the two data sets differed more around the time of maximum dynamic pressure with the maximum difference being about 90 psf.

Figure 18 shows how the Mach number predicted by the FADS system differed from the BET. As be seen in Fig. 18, both sets of data followed similar trends with the FADS data being less than the BET throughout this portion of the trajectory. The FADS and BET data differed by as much as 0.07 Mach and like $\mathrm{q}_{\mathrm{c}}$, both datasets differed the most around the time of maximum $\mathrm{q}_{\mathrm{c}}$.

Possibilities for the differences observed between the FADS and the BET data include the fact that the FADS calibration database did not include any wind-tunnel data. Also, the influence of the ACM and AM were not accounted for in the CFD data which was used for the FADS calibration database. Finally, CFD only modeled the portion of the vehicle forward of the AM nozzles. Since the PA-1 trajectory was entirely subsonic, the presence of the AM nozzles and the CM would influence the pressure distribution on the nosecone.

\section{ACM Jet Interaction with FADS Ports}

In an attempt to quantify the effect of the ACM on the nosecap FADS, a CFD analysis was performed. The data for this analysis was generated with the use of OVERFLOW; ${ }^{9}$ a Navier-Stokes flow solver for structured grids. Like the calibration data set, only the portion of the vehicle forward of the AM was modeled.

To perform the analysis, two sets of data were generated. One set of data modeled the ACM firing while the other set did not. Each set of data consisted of ten cases, which had input for PA-1 flight conditions from ten distinct instances in time along the PA- 1 flight trajectory. For all cases, input for $\alpha$ and $\beta$ were taken from the FADS results, while Mach input was taken from the BET, and $\mathrm{P}_{\infty}$ was provided by balloon data. In order to determine the nozzle boundary conditions for the ten ACM firing cases, ACM nozzle pintle position and ACM chamber pressure were taken from the Operational Flight Instrumentation (OFI) data set.

To understand how the change in pressure distribution due to the ACM firing affected the FADS output, surface pressures on the nosecap of the CFD model were extracted from locations with the same 
clock and cone angles as the vehicle. Then the groups of surface pressures extracted from each case were passed through the FADS algorithm to see how each case from the ACM-on set differed from the ACM-off set.

The percentage increase in pressure at the pressure ports due to the ACM firing is given by Eq. (17), and the values of $\Delta \mathrm{P}_{\mathrm{i}} \%$ for each port at the ten instances in time are illustrated in Fig. 19. Also illustrated in Fig. 19 is $\mathrm{q}_{\mathrm{c}}$ generated by the BET, and green lines showing when events of interest occurred during that portion of the trajectory.

$$
\Delta P_{i} \%=\frac{\left(P_{i}(\text { ACM on })-P_{i}(\text { ACM off })\right)}{P_{i}(A C M \text { off })} \times 100
$$

As can be seen in Fig. 19, there was minimal influence of the ACM at the center of the nosecap. However, as one moved away from the center towards the outer ports, the influence of the ACM became more pronounced with the ports on the outer circle being influenced the most. The differences increased as $\mathrm{q}_{\mathrm{c}}$ increased with a maximum difference $3.4 \%$.

Plots of the values of $\alpha, \beta$, Mach, $\mathrm{q}_{c}$, and $\mathrm{P}_{\infty}$ resulting from the CFD study are illustrated in Figs. 20 to 24. Each of these figures show the ten instances in time where input was taken to run the analysis. At each instance, the FADS output for the CFD case with the ACM on along with the FADS output for the case with the ACM off are plotted. The FADS data resulting from the CFD data is plotted along with the FADS output for the PA-1 trajectory and the BET output for the PA-1 trajectory to show how the trends in the CFD cases compared to the BET and the FADS data trends. Also plotted in each figure is the difference between the ACM-on data and the ACM-off data at each instance in time.

Figure 20 illustrates the results of the CFD study for $\alpha$. As can be seen in Fig. 20, the maximum difference between the ACM-on and the ACM-off data was less than $0.5^{\circ}$ before reorientation started. This was less than the maximum difference of $2^{\circ}$ between the PA-1 FADS and BET. Figure 21, which illustrates the results of the CFD analysis for $\beta$, shows similar results. There was good agreement between the ACM-off data and FADS $\alpha$ and $\beta$ data from the PA- 1 trajectory which was used to generate the CFD data.

Figure 22 shows the resulting $\mathrm{q}_{\mathrm{c}}$ from the CFD study. From Fig. 22, it can be seen that there is some influence of the ACM on the FADS output. However, the difference between the ACM-on and the ACM-off data is less than the difference between the FADS and the BET data. Similar to the PA-1 FADS and BET data, the maximum difference in the CFD data occurred around the time of maximum dynamic pressure, and this difference decreased until just after reorientation started.

Figure 23 shows the results of $\mathrm{P}_{\infty}$ from the CFD study. Again, it can be seen that there is some influence of the ACM on the FADS output. When compared to the PA-1 FADS and BET data, the overall difference between ACM-on and ACM-off data is less than the difference between the FADS and the BET data.

Figure 24 shows the results of Mach from the CFD study. Like $\mathrm{q}_{c}$ and $\mathrm{P}_{\infty}$, the CFD data followed similar trends to the PA-1 FADS and BET data, and overall the difference between the ACM-on and the ACM-off data was less than the difference between the PA-1 FADS and BET data.

Overall, the CFD study showed that the ACM motors did influence the FADS data. However, the magnitude of the influence of the ACM seen in the CFD data was less than the discrepancies between the PA-1 FADS and BET data. As mentioned previously, the calibration database of the FADS system was made up entirely of CFD data which only modeled the portion of the vehicle forward of the AM nozzles, and the ACM motor was not modeled. Also, the CFD analysis was done with the same geometry which was used for the calibration database. A follow on analysis would include replacing the CFD data in the FADS calibration database with CFD which modeled the entire vehicle and included effects of the AM and ACM motor. Then, this CFD analysis should be repeated with the updated geometry. 


\section{Conclusion}

A flush airdata sensing (FADS) system was tested on the nosecap of the Pad Abort-1 vehicle. All data was post processed to estimate values of angle of attack $(\alpha)$, angle of sideslip $(\beta)$, impact pressure $\left(\mathrm{q}_{\mathrm{c}}\right)$, freestream static pressure $\left(\mathrm{P}_{\infty}\right)$, and Mach number. The purpose of this system was to test whether or not reasonable data could be obtained from a FADS system if it were placed in close proximity to firing rocket motor nozzles like the attitude control motor (ACM) nozzles on PA-1. To gauge the performance of the FADS system, the resulting data was compared to data from the best estimated trajectory (BET). Overall, the FADS data followed similar trends to the BET, but the FADS results differed from the BET significantly. A CFD study was done in an attempt to understand the impact of the ACM nozzles on the FADS data. Given the magnitude of the differences between the FADS and the BET data from PA-1, the CFD study showed that the ACM had less of an impact on FADS data than was expected. More investigation is needed to understand why the FADS data and the BET data from the PA-1 trajectory differed by as much as they did.

\section{Figures}

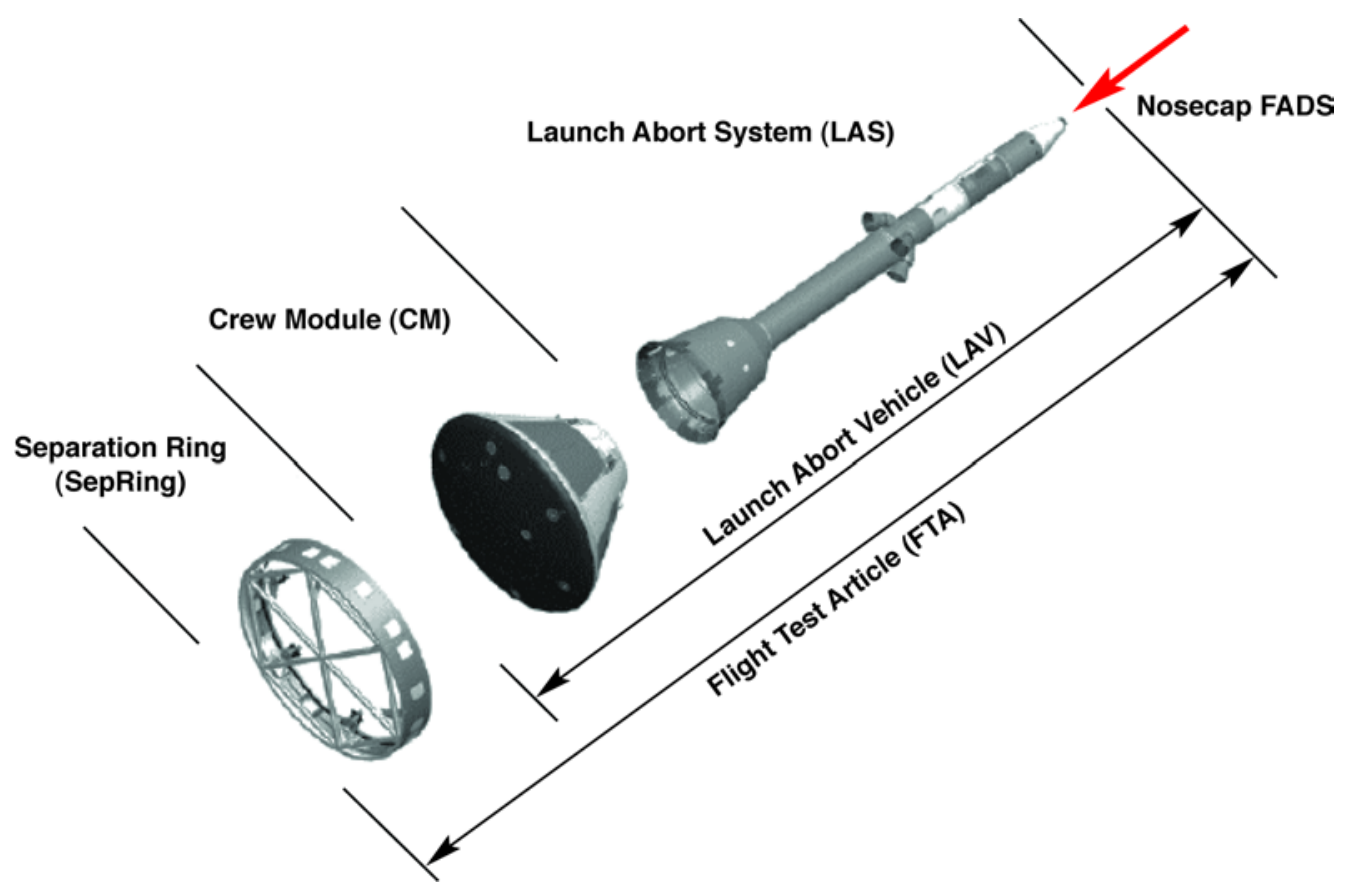

Figure 1. Basic layout of PA-1 Flight Test Article. 


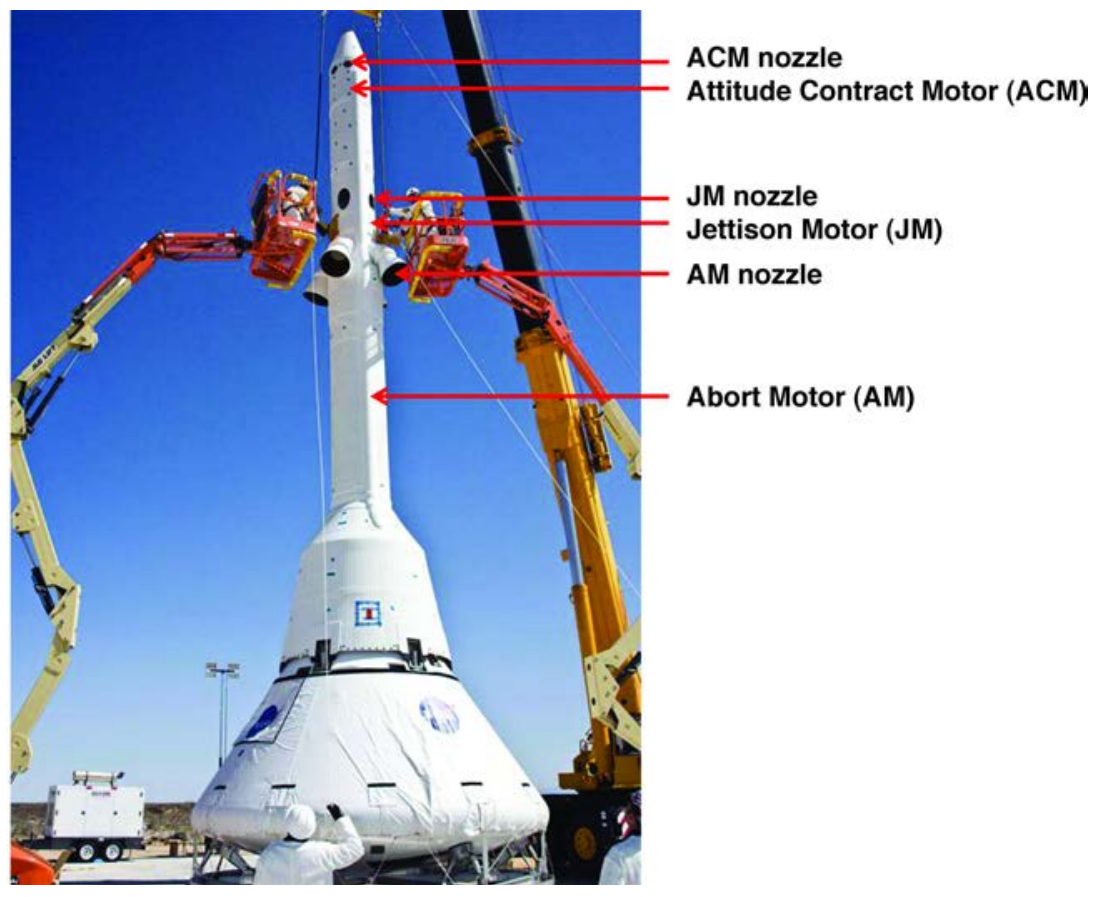

Figure 2. Location of motor nozzles.

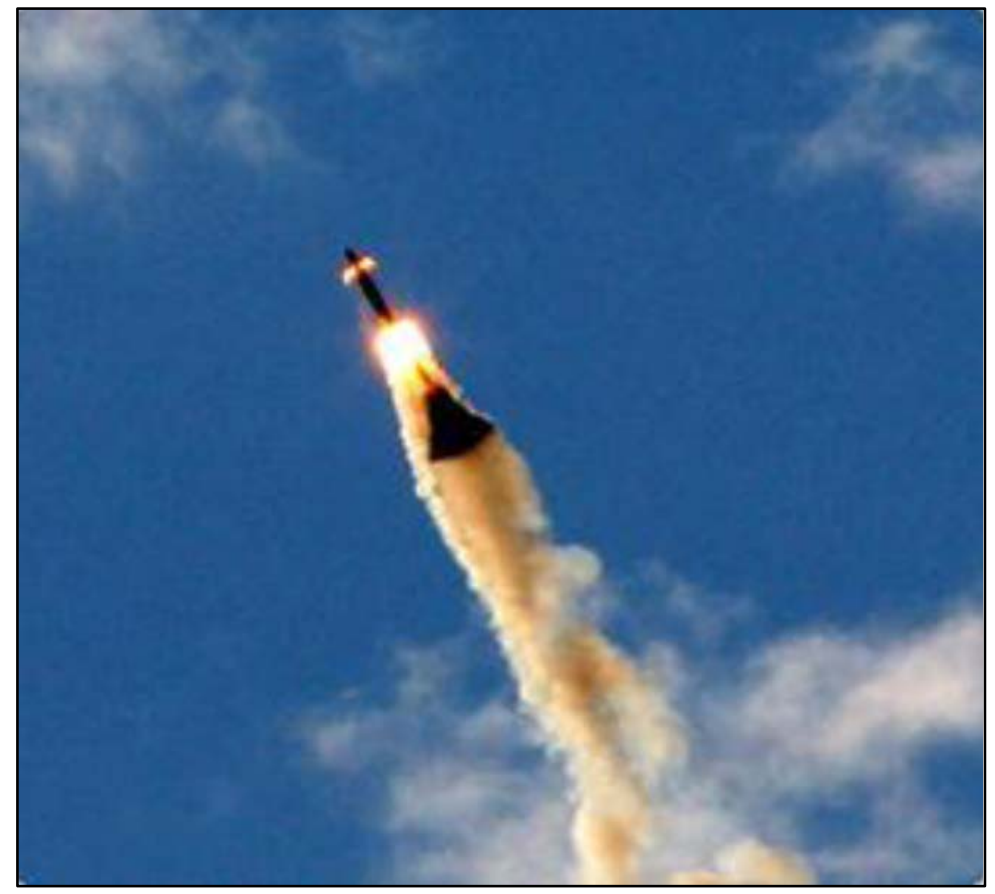

Figure 3. AM and ACM firing. 


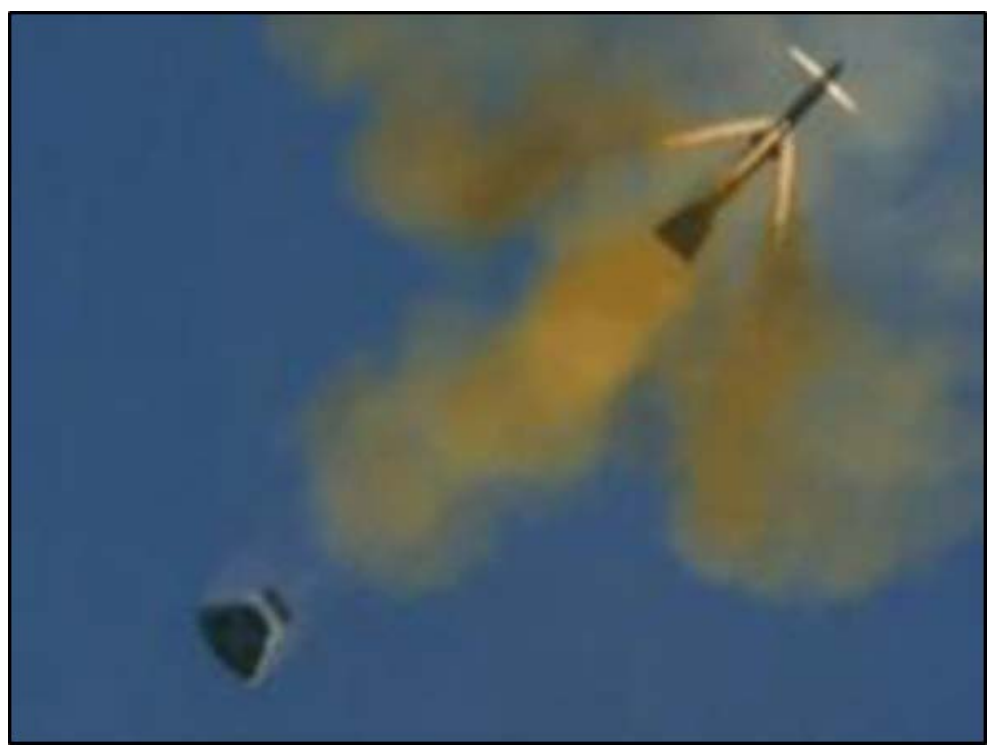

Figure 4. LAS jettison with JM and ACM firing.

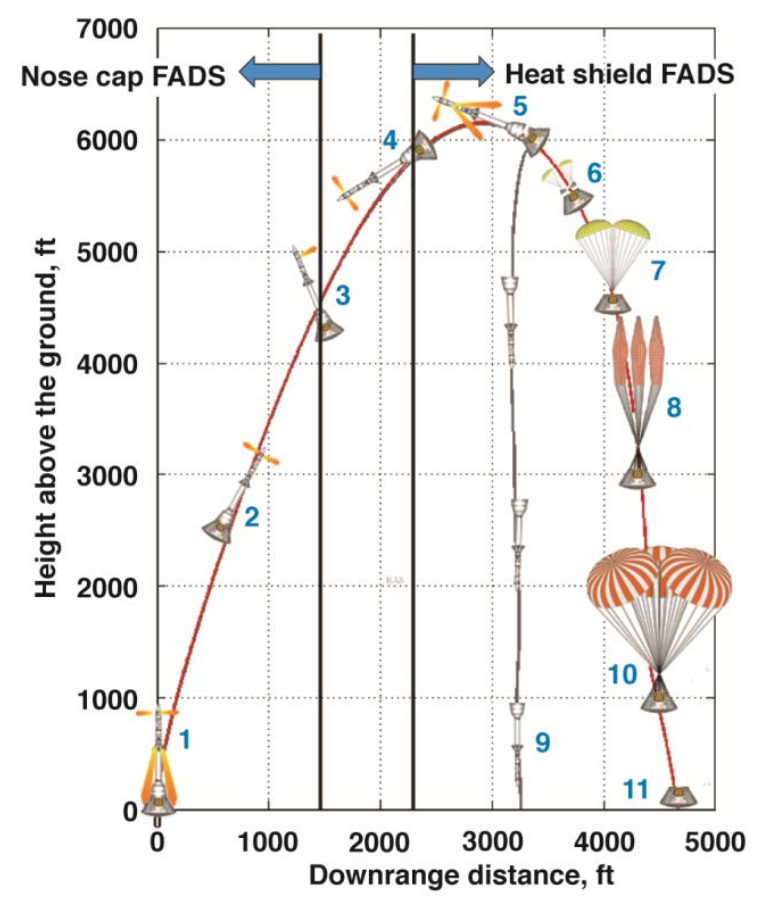

Figure 5. PA-1 trajectory. 


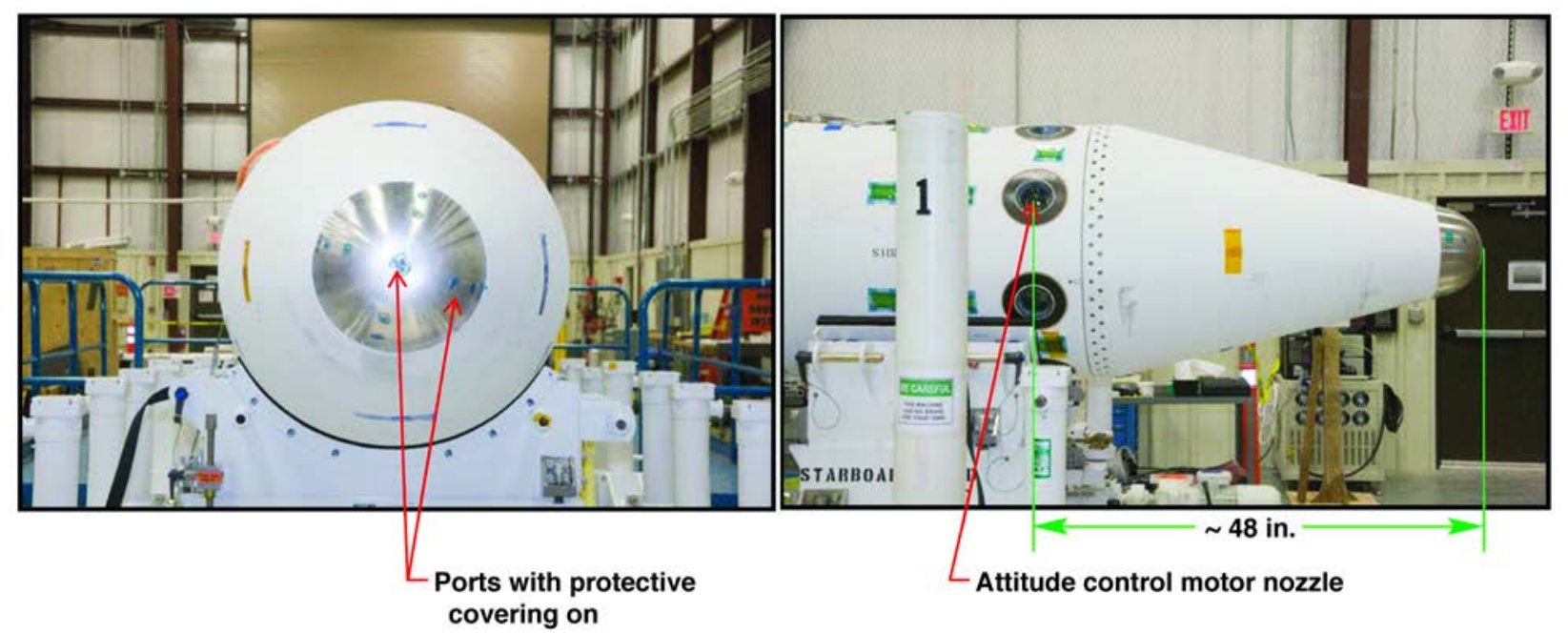

Figure 6. Location of FADS ports on LAS nosecap relative to all three motors on LAS.

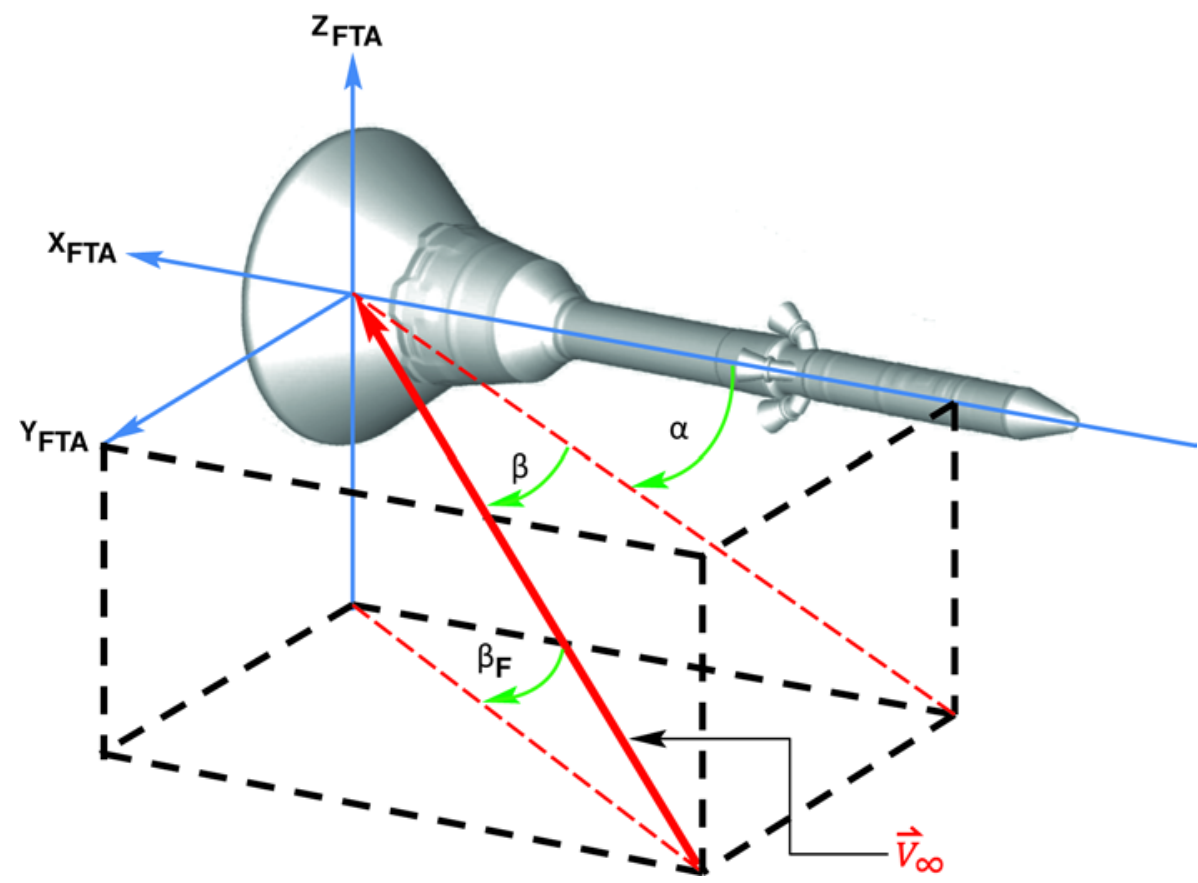

Figure 7. Illustration of FTA coordinate frame, angle of attack $(\alpha)$, sideslip $(\beta)$, and flank angle $\left(\beta_{F}\right)$. 


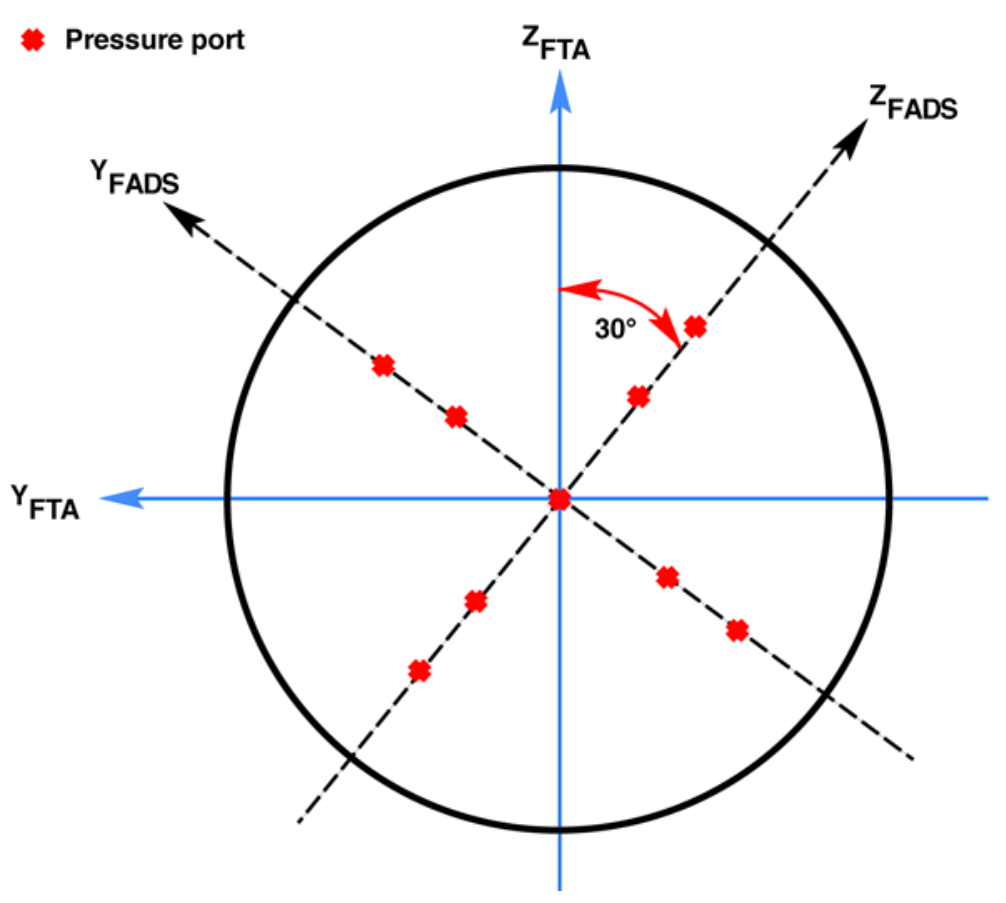

Figure 8. Illustration of nosecap FADS reference frame and FTA reference frame.
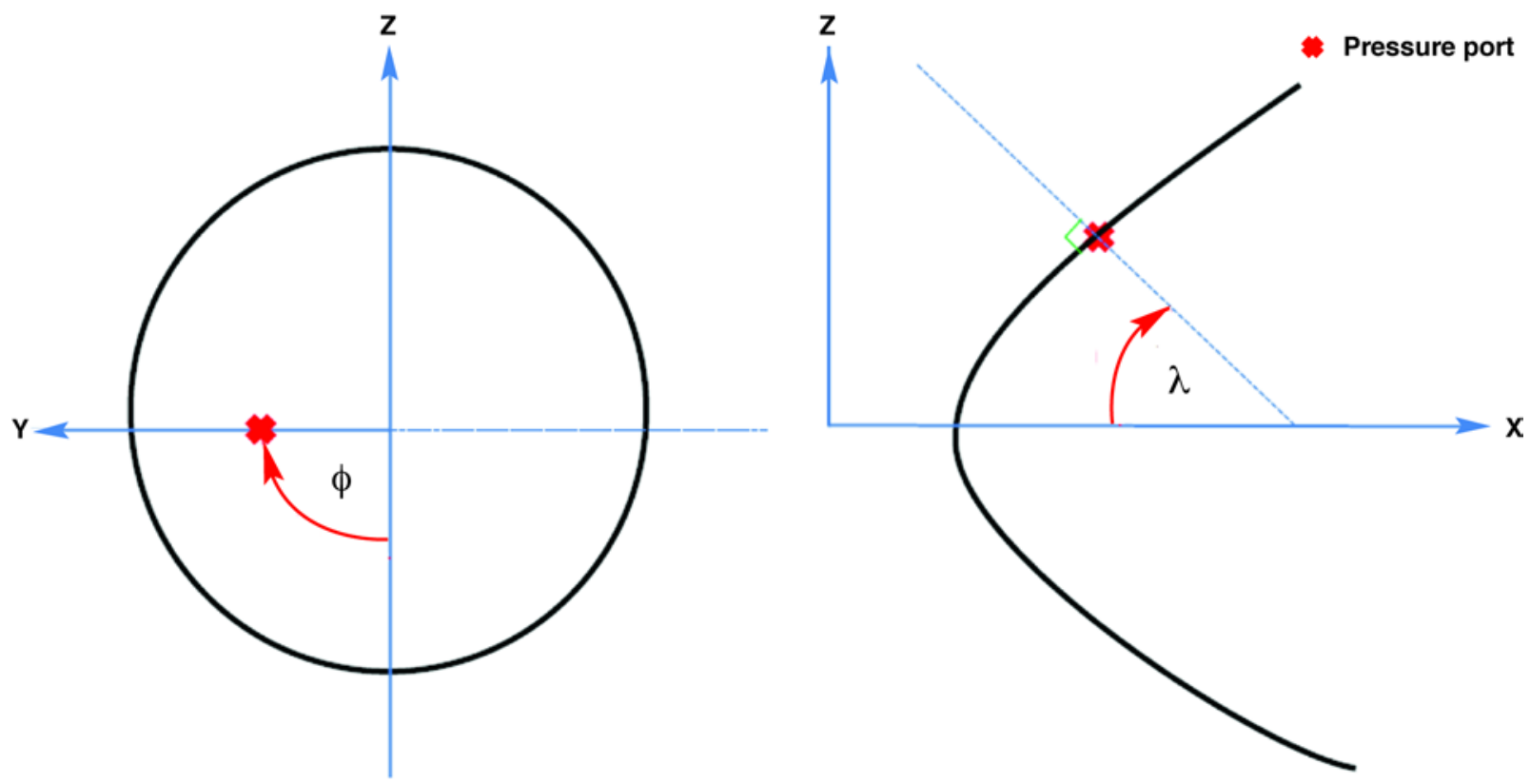

Figure 9. Definition of clocking angle $(\phi)$ and cone angle $(\lambda)$. 


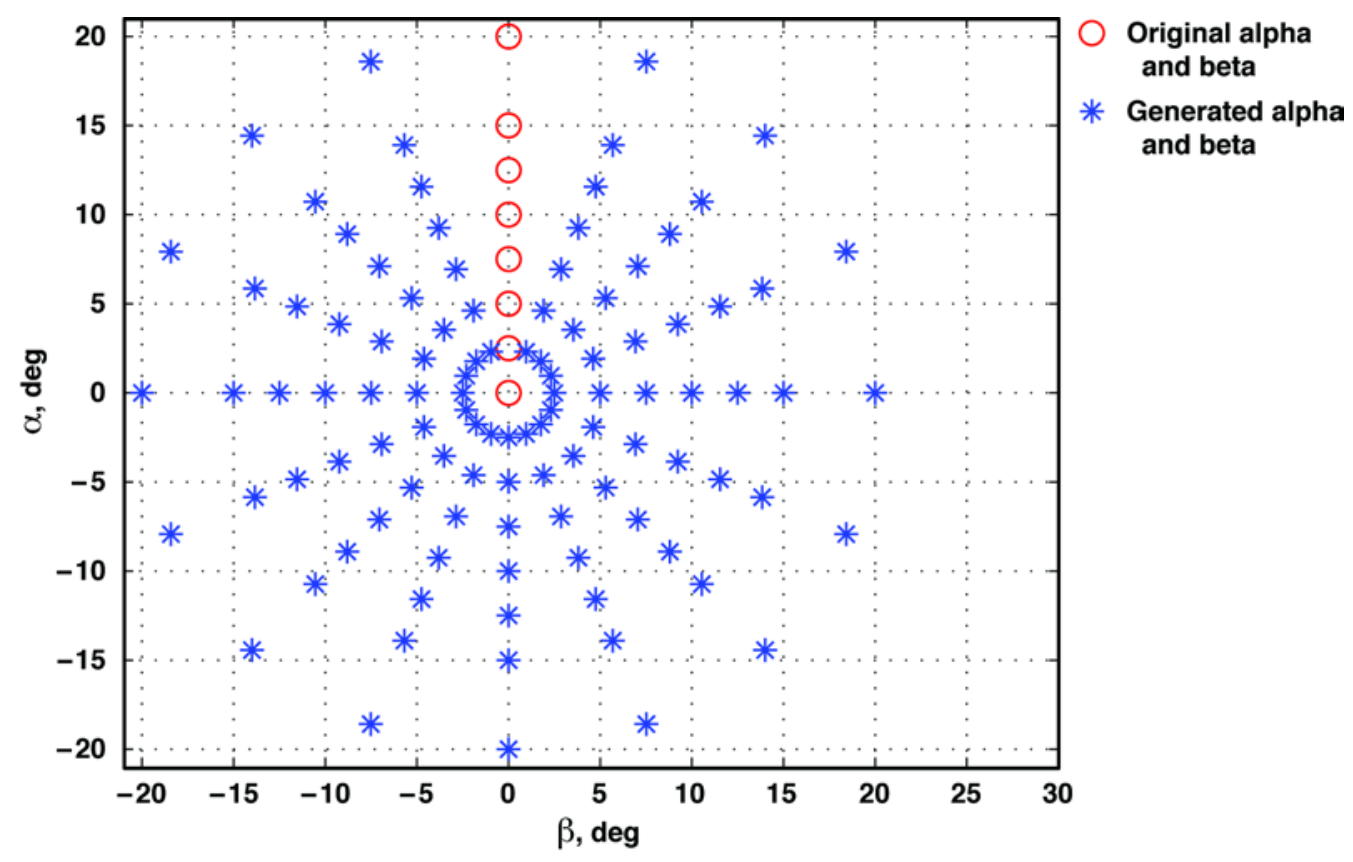

Figure 10. Range of $\alpha$ and $\beta$ for nosecap calibration data base.

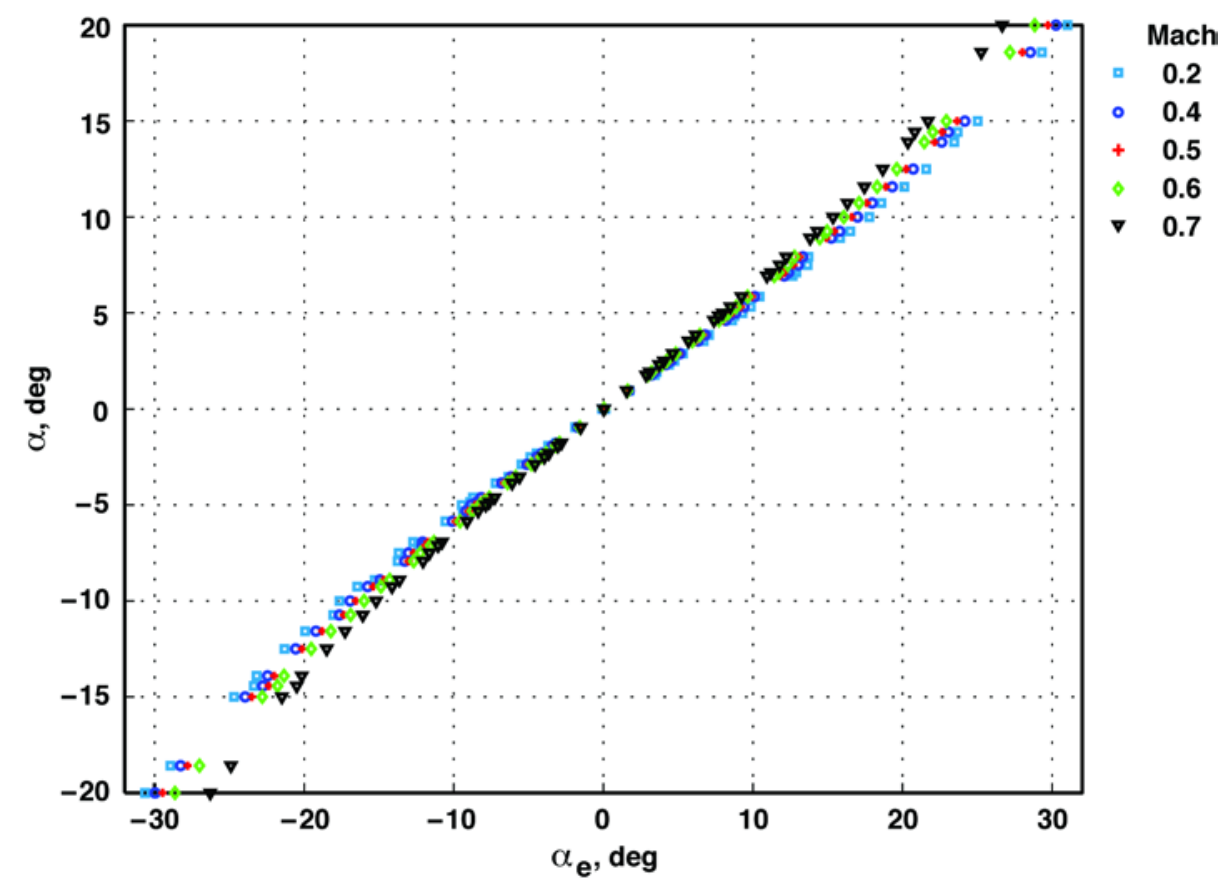

Figure 11. Calibration data for $\alpha$ at zero $\beta_{\mathrm{Fe}}$. 


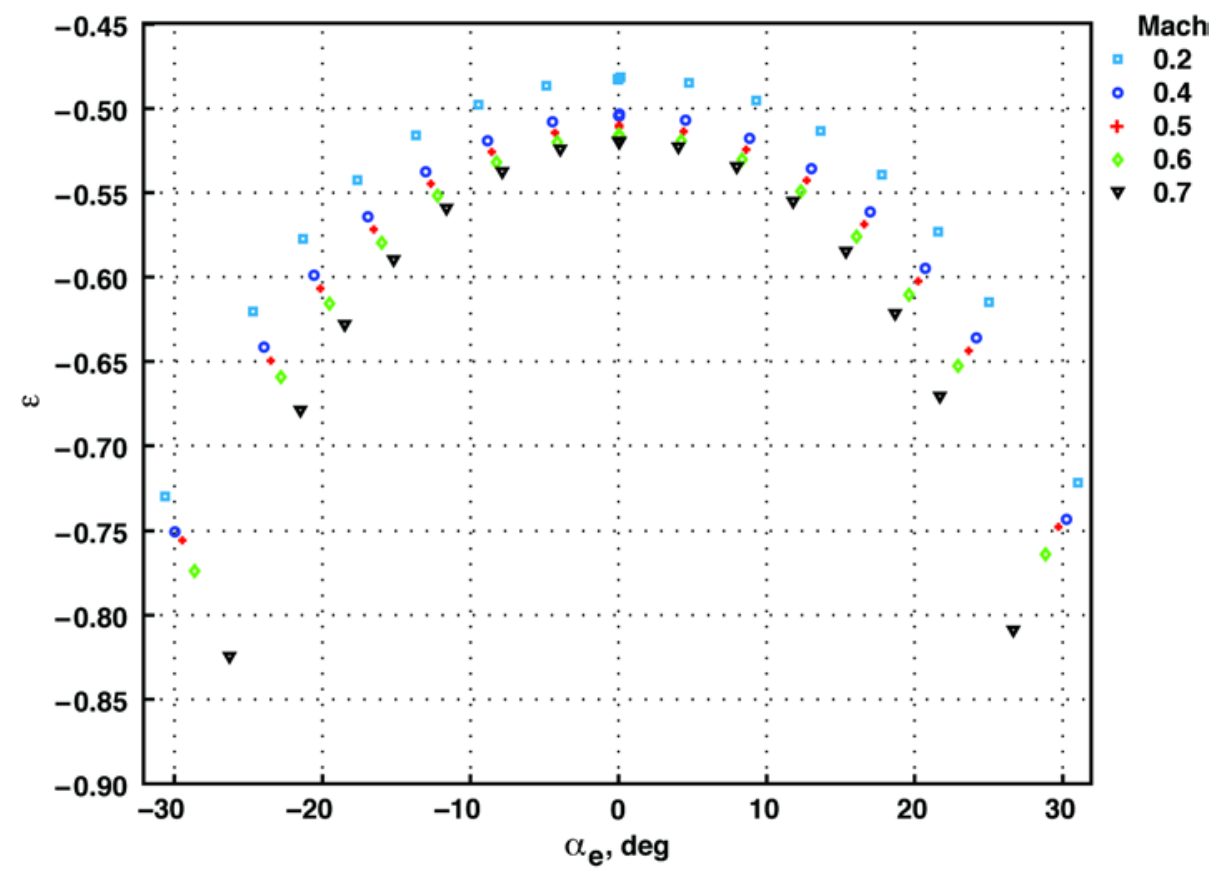

Figure 12. Calibration parameter $(\epsilon)$ versus $\alpha_{\mathrm{e}}$ at zero $\beta_{\mathrm{Fe}}$.

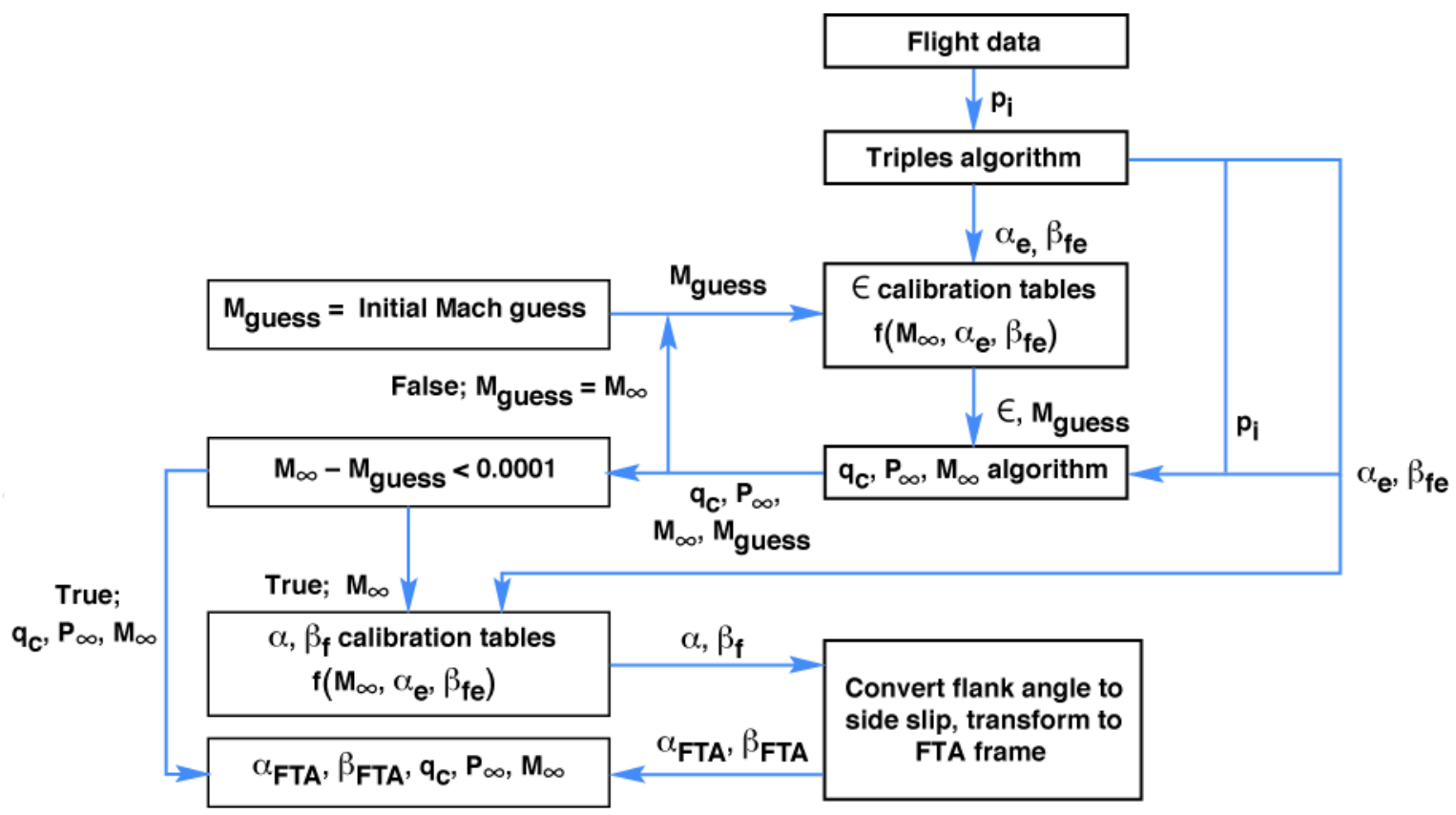

Figure 13. Nosecap FADS algorithm flow chart. 

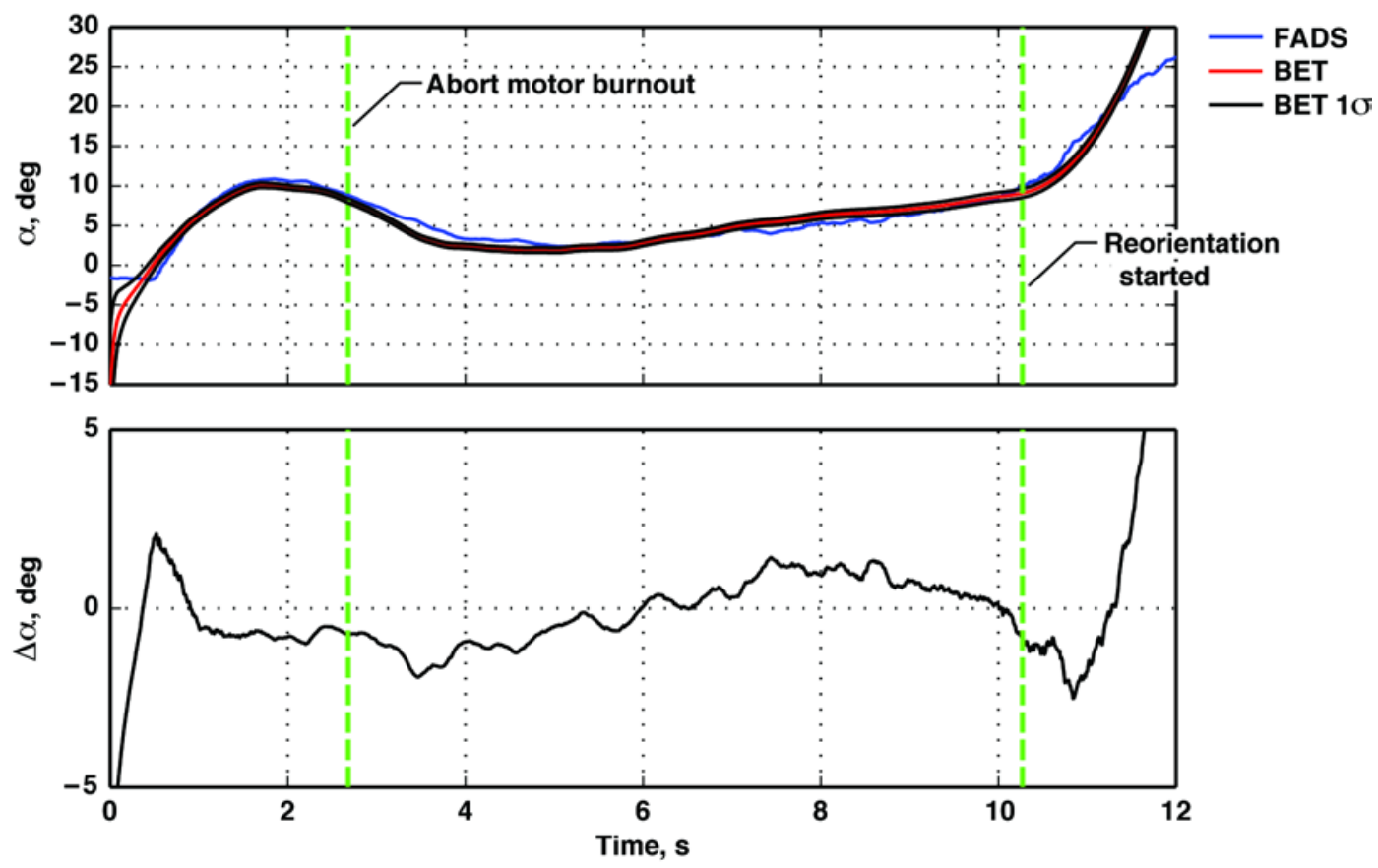

Figure 14. Comparison of FADS $\alpha$ to BET.
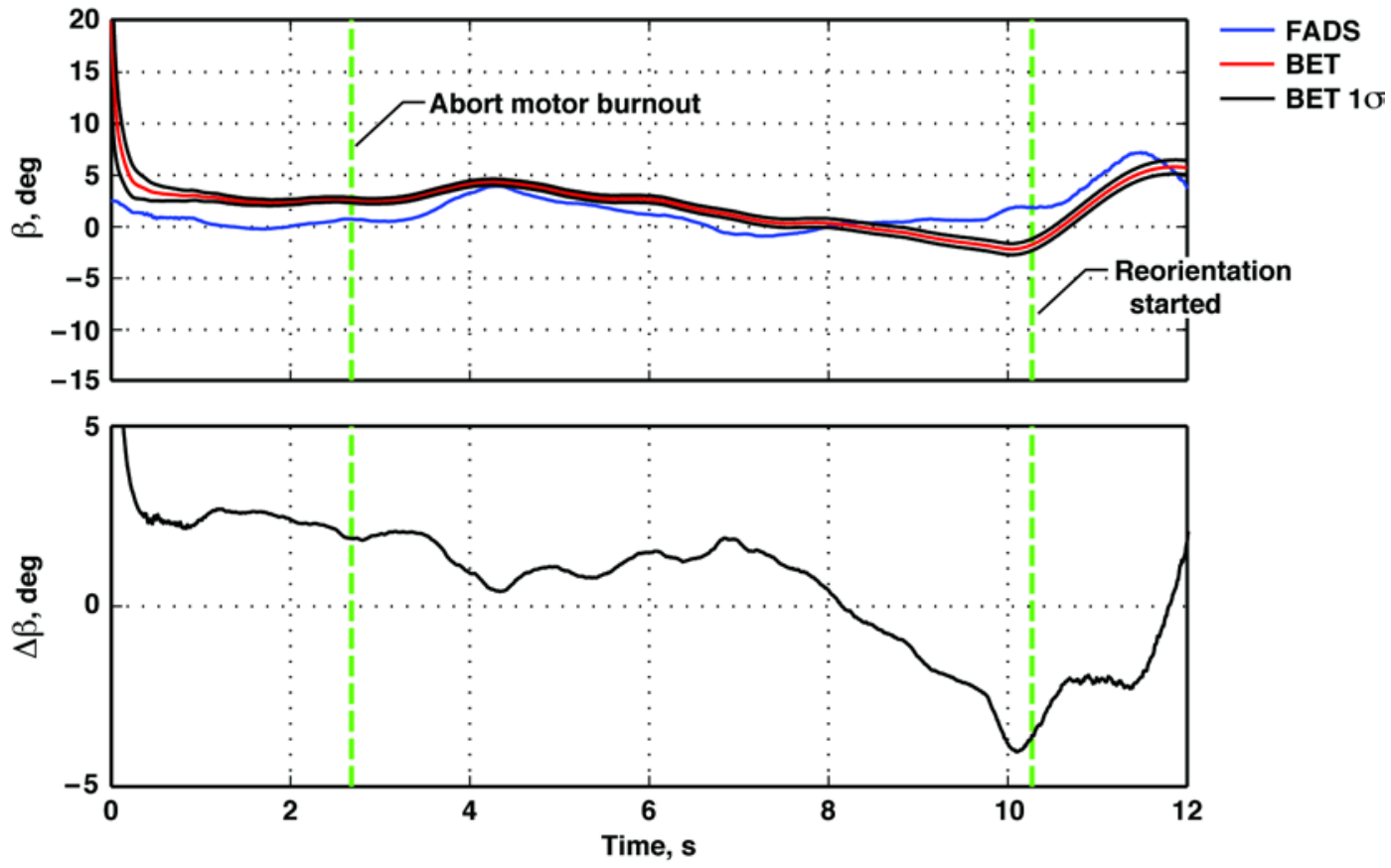

Figure 15. Comparison of FADS $\beta$ to BET. 

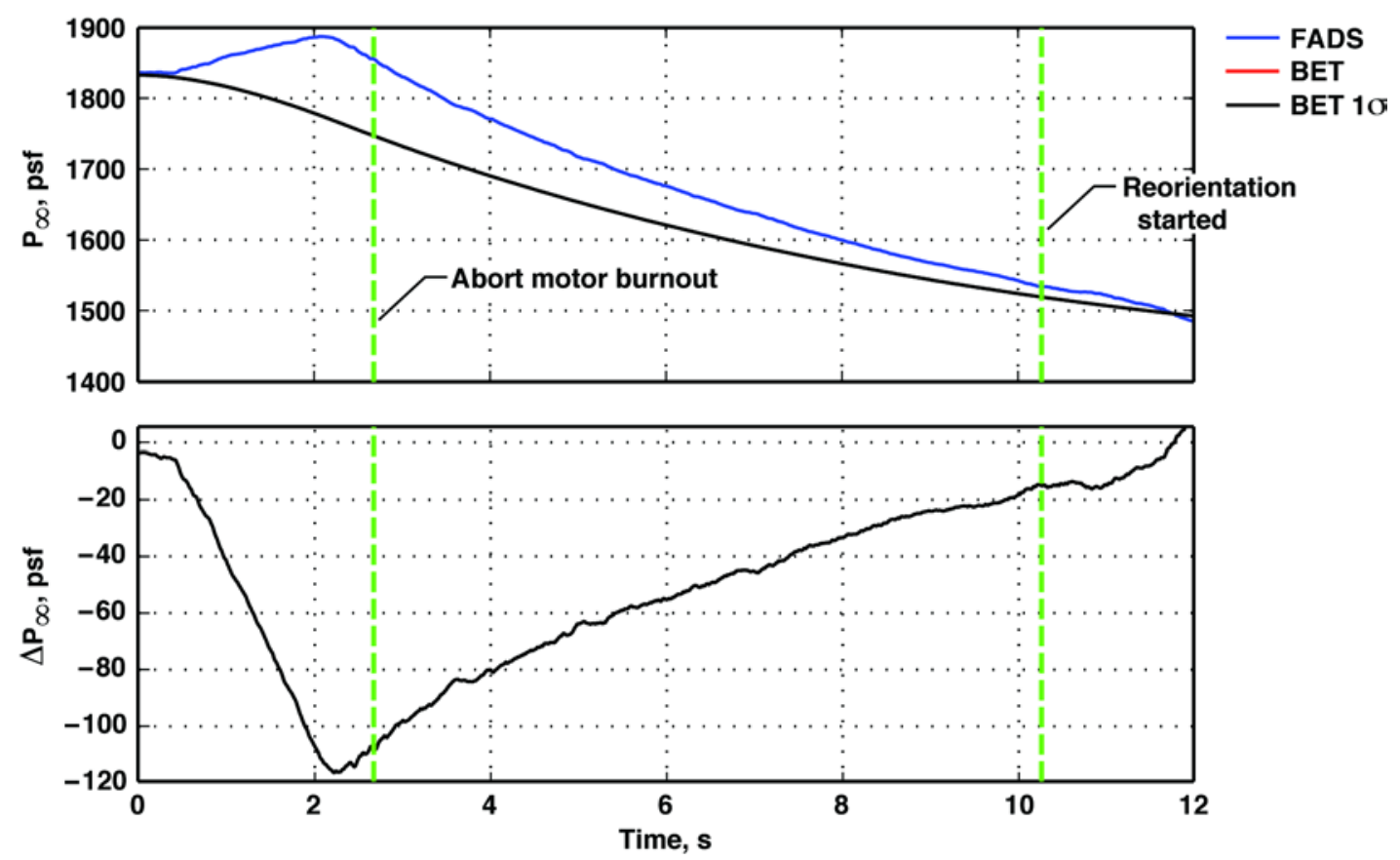

Figure 16. Comparison of FADS $P_{\infty}$ to BET.
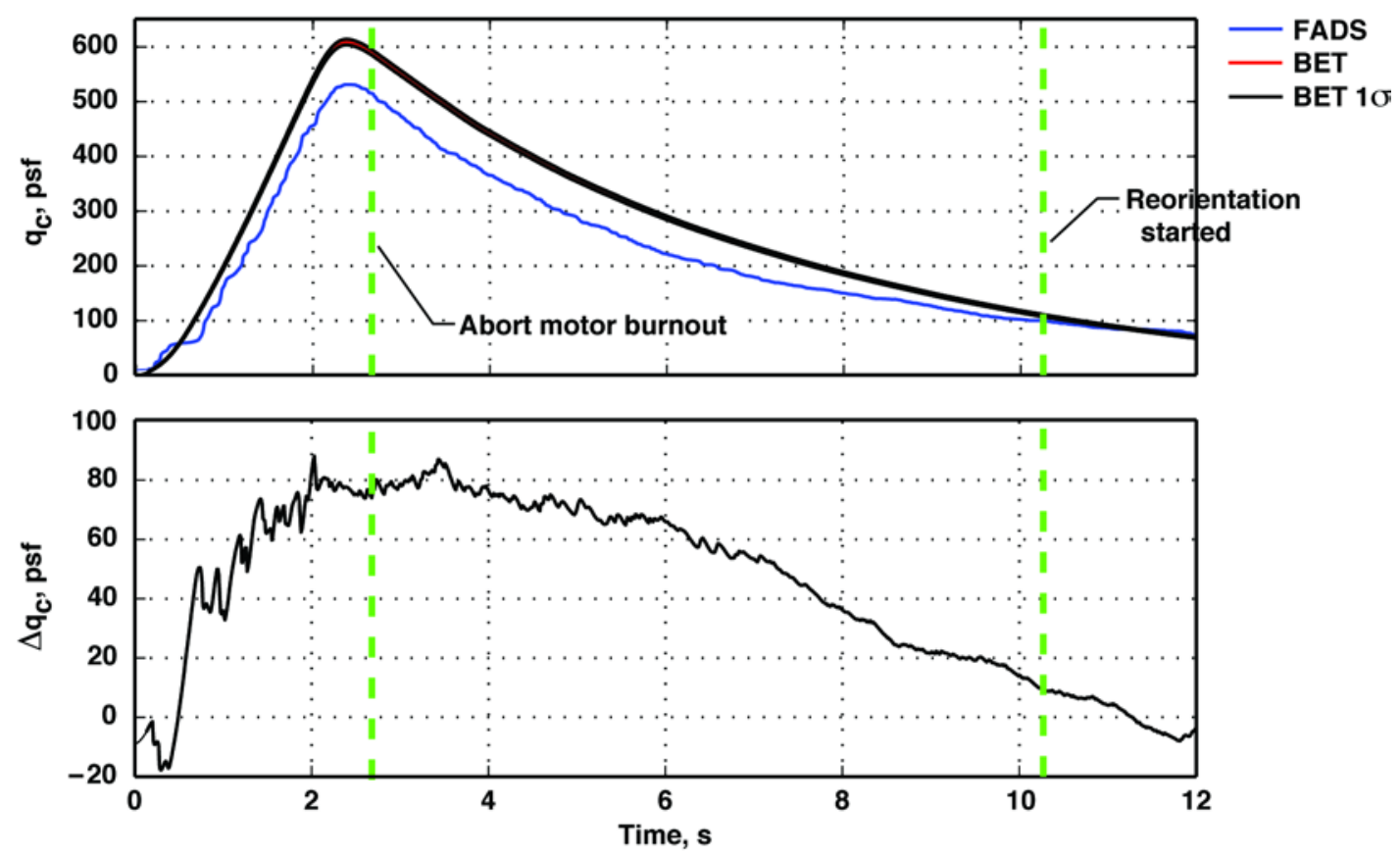

Figure 17. Comparison of FADS $q_{c}$ to BET. 

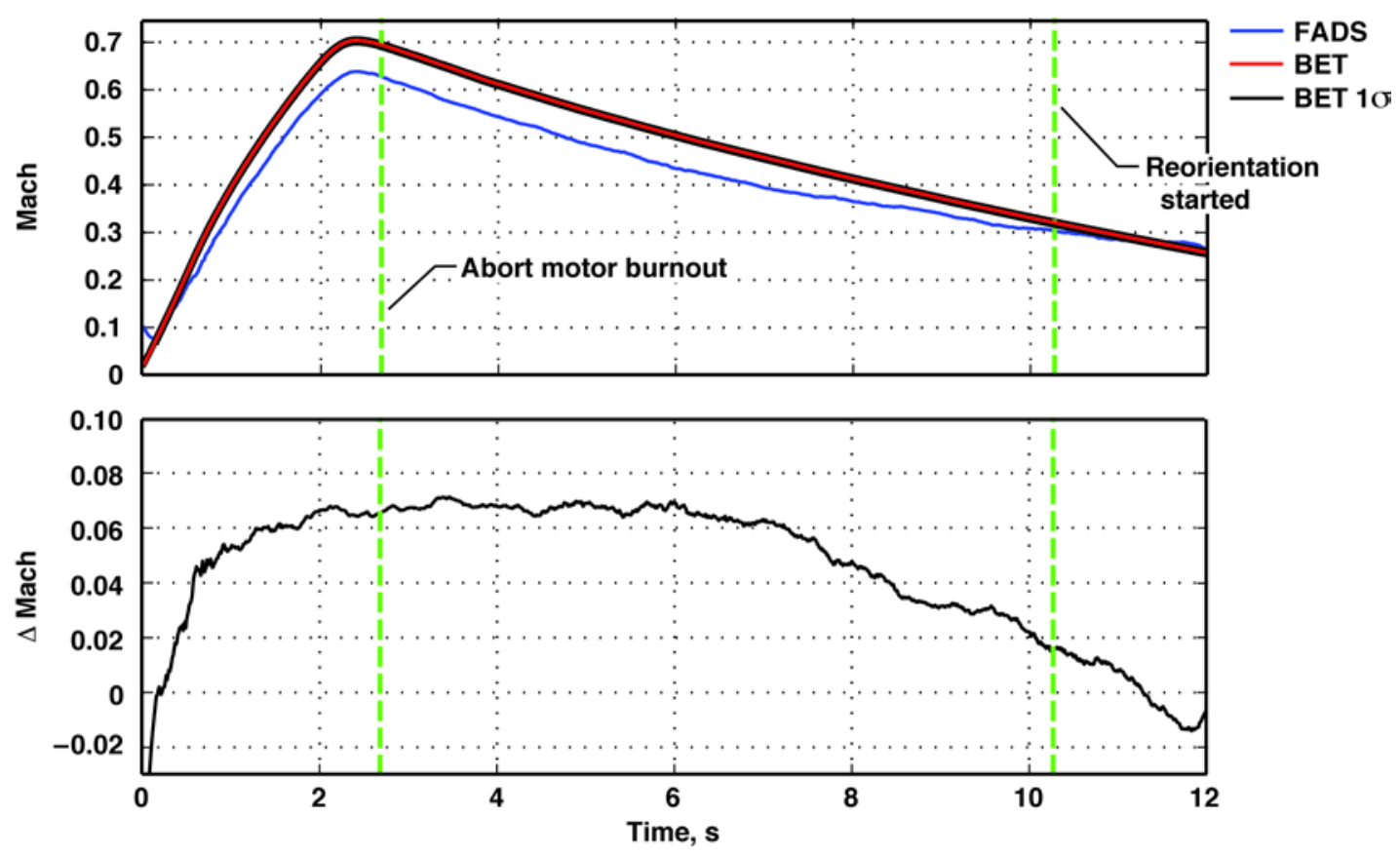

Figure 18. Comparison of FADS Mach to BET.

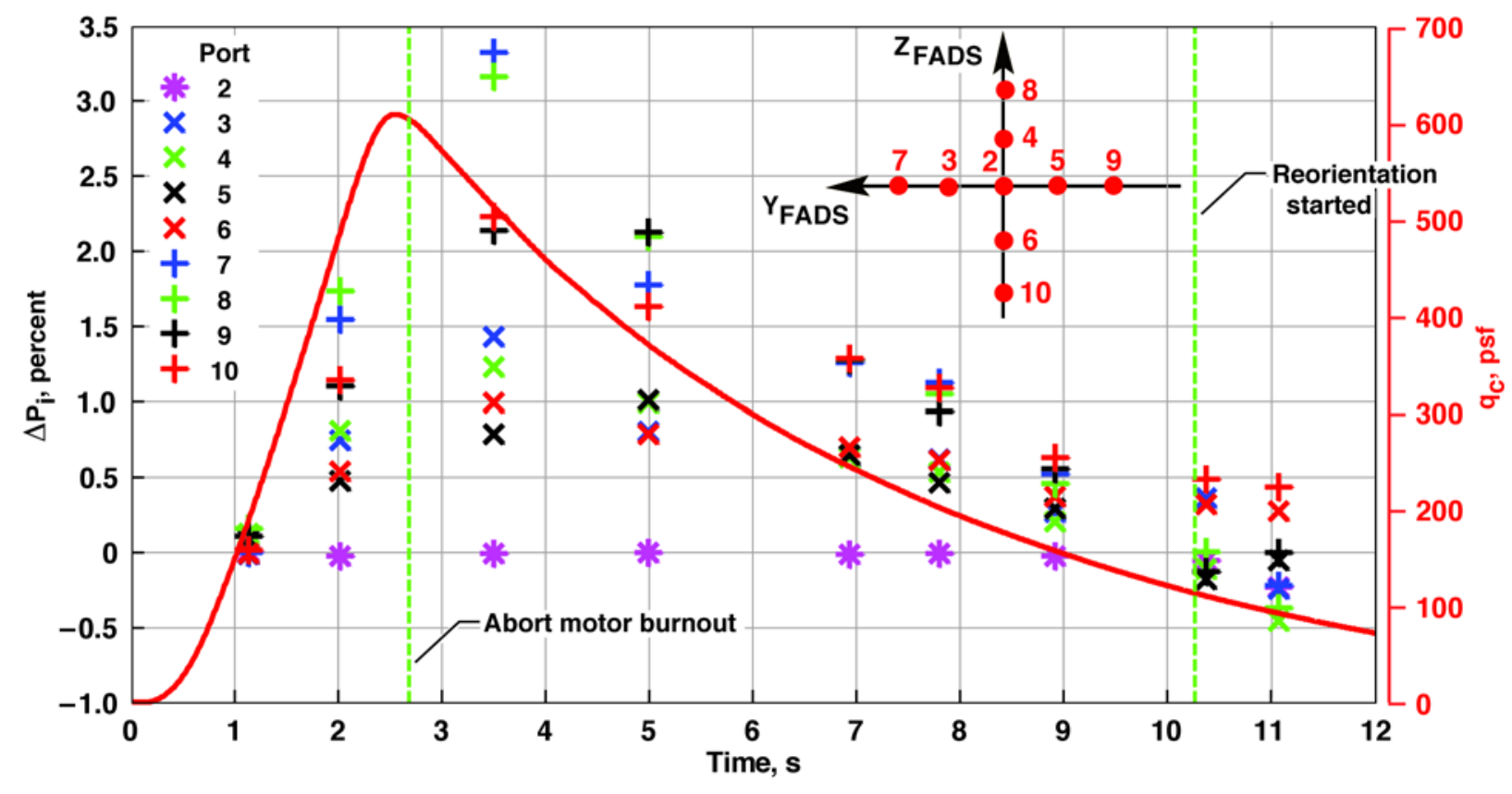

Figure 19. Plot of $q_{c}$ and percent change in pressure due to ACM at FADS ports from CFD study. 


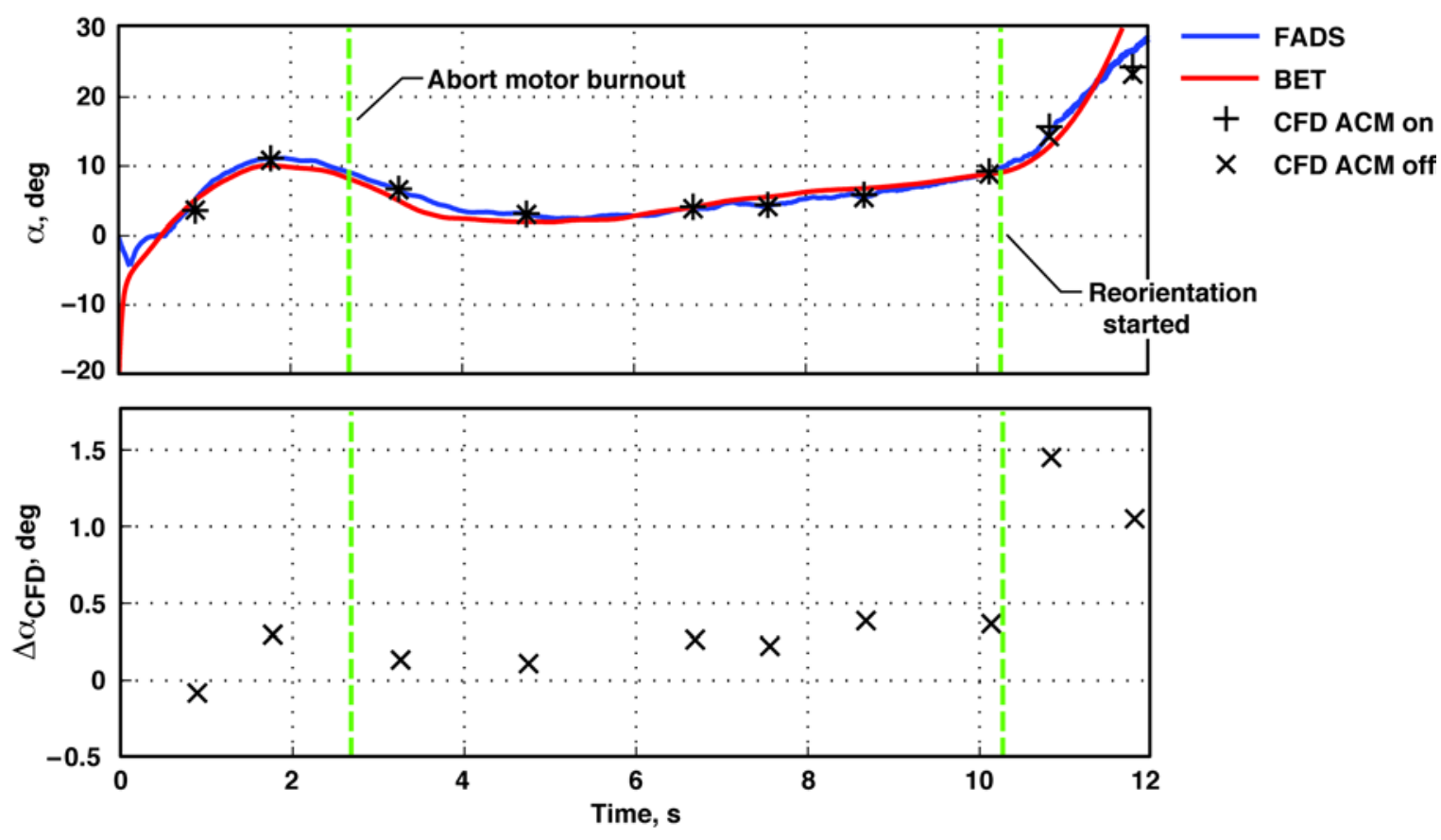

Figure 20. Comparison of ACM on to ACM off $\alpha$.
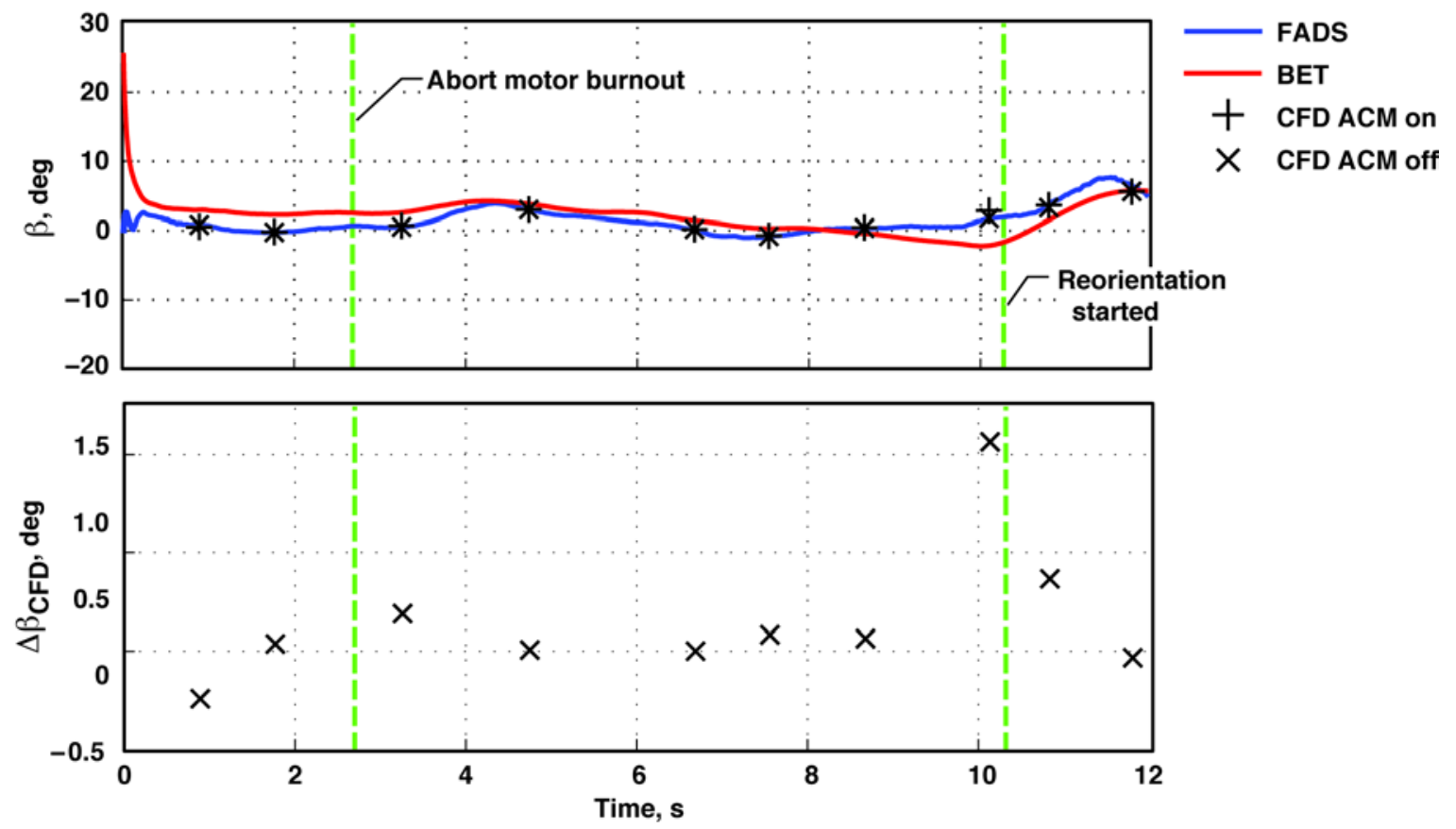

Figure 21. Comparison of ACM on to ACM off $\beta$. 

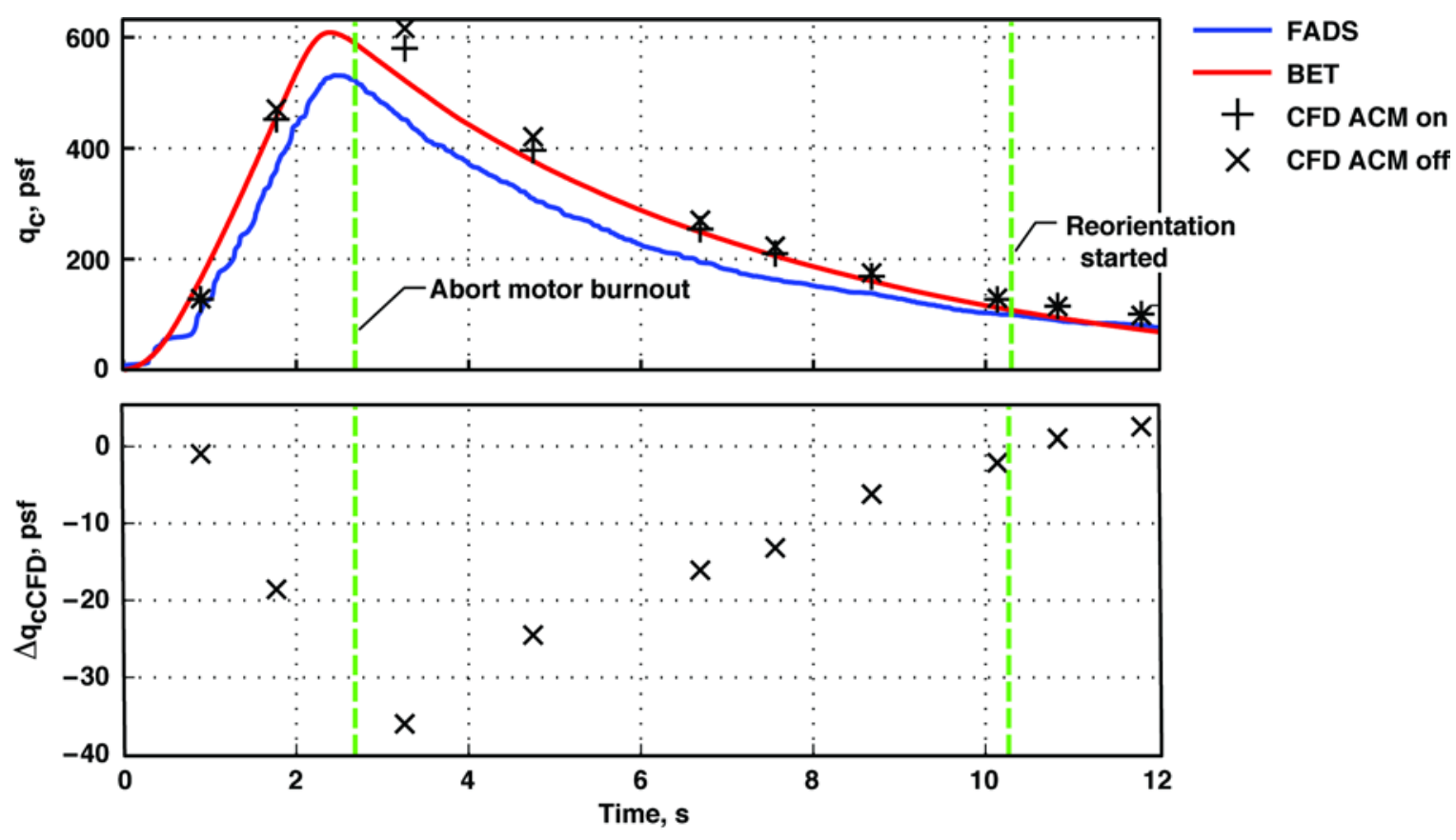

Figure 22. Comparison of ACM on to ACM off $\mathbf{q}_{c}$.
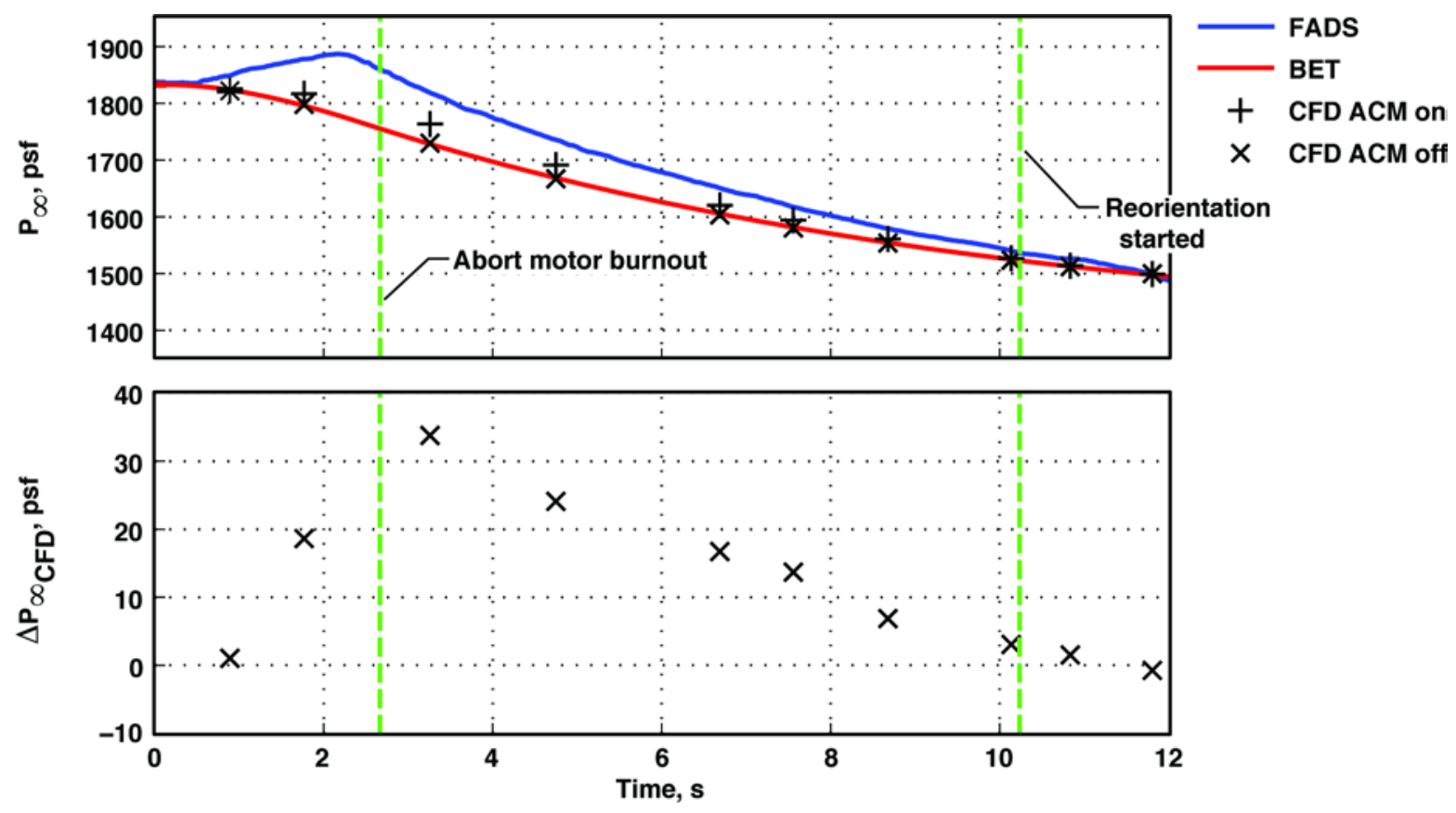

Figure 23. Comparison of ACM on to ACM off $P_{\infty}$. 

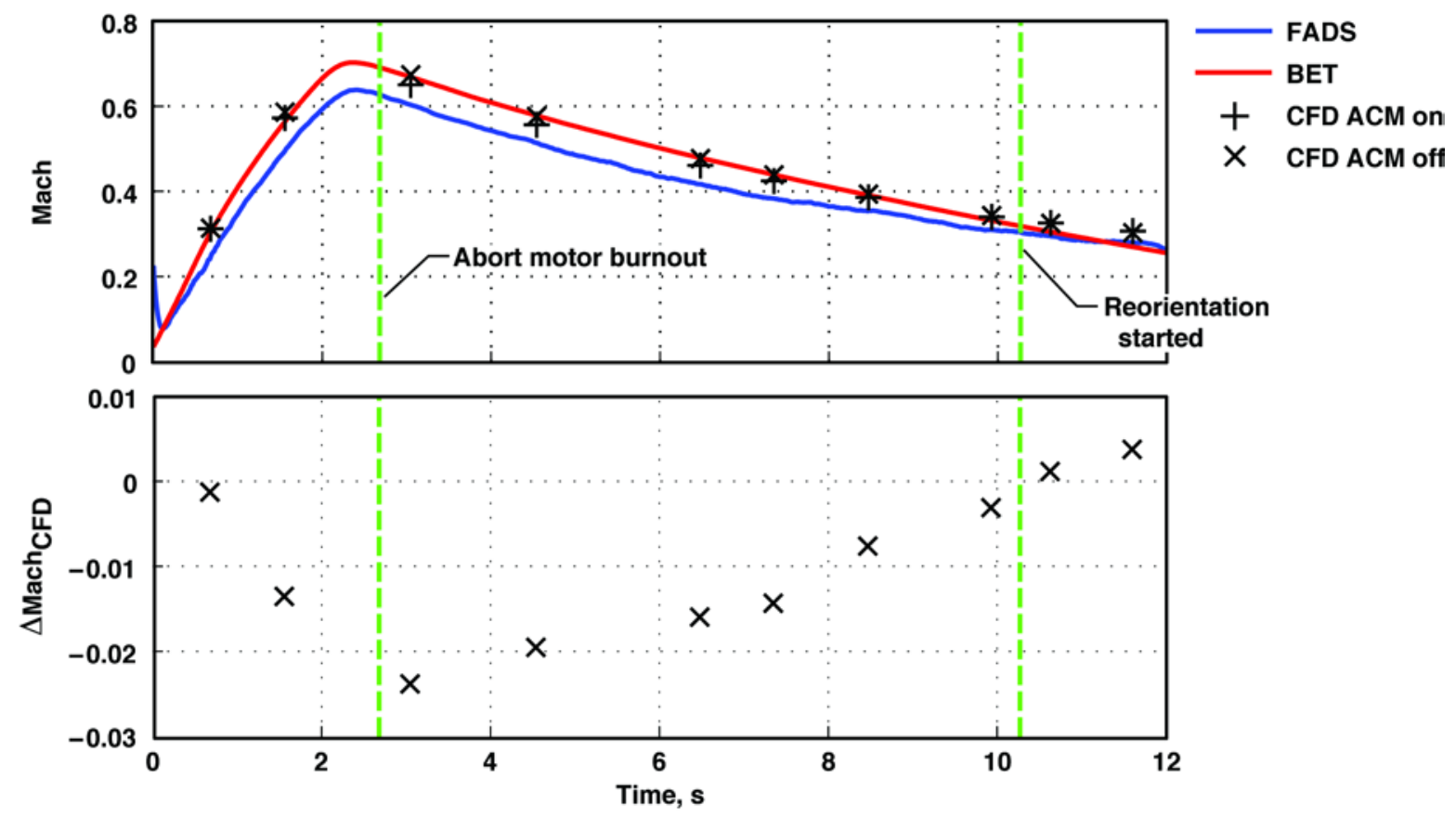

Figure 24. Comparison of ACM on to ACM off Mach.

\section{Acknowledgments}

The authors are grateful to Thomas M. Booth (NASA-JSC), James S. Greathouse (NASA-JSC), Darby Vicker (NASA-JSC), and Phillip C. Stuart (NASA-JSC) for providing CFD and analysis support for this work.

\section{References}

${ }^{1}$ Cary, J. P., and Keener, E. R., "Flight Evaluation of the X-15 Ball-Nose Flow-Direction Sensor as an Air-Data System,” NASA TN D-2923, 1965.

${ }^{2}$ Baumann, E., Pahle, J. W., and Davis, M. C., “X-43A Flush Airdata Sensing System Flight-Test Results,” Journal of Spacecraft and Rockets, Vol. 47, No. 1, 2010, pp. 48-61.

${ }^{3}$ Whitmore, S. A., Davis R. J., and Fife, J. M., "In-Flight Demonstration of a Real-Time Flush Airdata Sensing (RT-FADS) System,” NASA TM-104314, 1995.

${ }^{4}$ Ali, A. N., and Borrer, J., "PA-1 Flush Airdata Sensing Systems," Proceedings of the 49th AIAA Aerospace Sciences Meeting including the New Horizons Forum and Aerospace Exposition, Orlando, Florida, 4-7 January 2011.

${ }^{5}$ Jones, D. S., and Koelfgen, S. J., "Executive Summary of Propulsion on the Orion Abort Flight-Test Vehicles," AIAA 2012-3891, 2012.

${ }^{6}$ Idicula, J., Williams-Hayes, P. S., Stillwater, R., and Yates, Lt. M., “A flight Dynamics Perspective of the Orion Pad Abort One Flight Test,” AIAA 2009-5730, 2009.

${ }^{7}$ Kutty, P., Noonan, M., Karlgaard, C., and Beck R., “The Best Estimated Trajectory Analysis for Pad Abort One,” Proceedings of the 49th AIAA Aerospace Sciences Meeting, Orlando, Florida, 4-7 January 2011.

${ }^{8}$ Whitmore, S. A., Cobleigh, B. R., and Haering, E. A., "Design and Calibration of the X-33 Flush Airdata Sensing (FADS) System,” NASA/TM-1998-206540, 1998.

${ }^{9}$ Nichols, R. H., Buning, P. G., “User’s Manual for OVERFLOW 2.2,” URL: http://people.nas.nasa.gov/ pulliam/Overflow/ Overflow_Manuals.html, [cited 22 July, 2013]. 